\title{
The Correlation between X-ray and UV Properties of BAL QSOs
}

\author{
LuLu Fan, ${ }^{1,3}$ HuiYuan Wang, ${ }^{1,2}$ Tinggui Wang, ${ }^{1,2}$ Junxian Wang, ${ }^{1,2}$ Xiaobo Dong, ${ }^{1,2}$ Kai \\ Zhang, ${ }^{1,2}$ Fuzhen Cheng, ${ }^{1,2}$
}

\begin{abstract}
We compile a large sample of broad absorption lines (BAL) quasars with X-ray observations from the XMM-Newton archive data and Sloan Digital Sky Survey Data Release 5. The sample consists of 41 BAL QSOs. Among 26 BAL quasars detected in X-ray, spectral analysis is possible for twelve objects. X-ray absorption is detected in all of them. Complementary to that of Gallagher et al. (2006) (thereafter G06), our sample spans wide ranges of both BALnicity Index $(\mathrm{BI})$ and maximum outflow velocity $\left(v_{\max }\right)$. Combining our sample with G06's, we find very significant correlations between the intrinsic X-ray weakness with both BALnicity Index (BI) and the maximum velocity of absorption trough. We do not confirm the previous claimed correlation between absorption column density and broad absorption line parameters. We tentatively interpret this as that X-ray absorption is necessary to the production of the BAL outflow, but the properties of the outflow are largely determined by intrinsic SED of the quasars.
\end{abstract}

Subject headings: quasars: absorption lines - X-rays: general

\section{INTRODUCTION}

About 10\%-30\% of optically selected QSOs show broad absorption lines (BAL) in their UV spectra, indicative of outflows with velocities up to 0.1c(Hewett \& Foltz 2003; Reichard et al. 2003). The similarity in the UV continuum and emission lines between BAL and non-BAL QSOs suggests that BAL QSOs are otherwise normal QSOs viewed in the direction covered by the outflow (e.g. Weymann et al. 1991). One exception to these similarities is that BAL QSOs are soft X-ray faint compared to non-BAL QSOs (e.g. Green et al. 1995; Brinkmann et al. 1999). The weakness in X-rays is interpreted as due to strong absorption

\footnotetext{
${ }^{1}$ Center for Astrophysics, University of Science and Technology of China, Hefei, 230026, China;

${ }^{2}$ Joint Institute of Galaxies and Cosmology, USTC and SHAO, CAS

${ }^{3}$ SISSA/ISAS,Via Beirut 2-4,I-34014 Trieste,Italy
} 
rather than intrinsic difference. Evidence for this has been accumulated now from detailed studies of X-ray spectra of a few bright BAL quasars, which display X-ray absorption with column densities from $10^{22}$ to $\geq 10^{24} \mathrm{~cm}^{-2}$ (Wang et al. 1999; Gallagher et al. 1999, 2002).

Giving the ubiquity of X-ray absorption in BAL quasars, it is natural to ask whether and how the X-ray absorbing gas is connected to the UV BAL phenomenon. It has been known for quite long time that BAL gas should be either confined into small clumps or shielded from the intense soft X-rays in order to match the observed profile. Murray et al. (1995) proposed that the highly ionized gas at the base of disk wind (shielding gas) can naturally filter the soft X-ray radiation to prevent the gas to be over-ionized so that the radiative acceleration is effective (See also Proga et al. 2000). As both UV and X-ray absorbers are part of the continuous outflow, the column densities of the two are expected to be correlated. Indeed, Brandt,Laor \& Wills (2000) identified a correlation between the equivalent width of C IV absorption line and the soft X-ray weakness in a sample of bright quasars, including half a dozen BAL QSOs.

Wang et al. (2005, 2007) found that electron scattering of the shielding gas can explain the distribution of continuum polarization in quasars, and the resonant scattering of BAL outflow can explain the observed polarized spectrum of BAL. They further noted that certain special features should appear in the polarized spectrum if the size of the shielding gas is comparable with that of the BAL outflows. As these features are only found in several low-ionization BAL (LoBAL) QSOs (see their paper for details and also Ogle et al. 1999), the shielding gas is likely well inside the BAL outflow except in LoBAL QSOs. A similar conclusion has been reached by studying the X-ray spectra of BAL QSOs (e.g. Gallagher et al. 2004, 2006). On the other hand, as pointed by Wang et al. (2000), in order to keep sufficient opacity in the soft X-ray between $0.2-0.3 \mathrm{keV}$, the absorber must have large column density also of Li-like ions because those ions are responsible for both the soft X-ray absorption between $0.2-0.3 \mathrm{keV}$ and the high ionization UV BALs. Though this band is notoriously difficult to be studied, they argued that at least in three bright low redshift BAL QSOs, the $\mathrm{X}$-ray absorption opacity around $0.2-0.3 \mathrm{keV}$ is large, suggesting very large column density of Li-like ions. However, a relatively small fraction of X-ray absorbing gas at moderate ionization level will be sufficient to suppress the soft X-ray flux.

If the X-ray shielding is critical to the ionization balance in the BAL outflow, which in turn affects the radiative accelerating force on the outflow, one would expect that kinematic properties and column density of BAL outflow will somehow correlate with the properties of the X-ray absorber. In a sample of BAL quasars observed by Chandra, G06 found a weak correlation between the maximum outflow velocity $\left(v_{\max }\right)$ of BAL and the indicator $\left(\Delta \alpha_{\text {ox }}\right)$ of X-ray absorption. Their finding agrees with the qualitative analysis that the strong soft 
X-ray absorption leads to more Li-like ions, thus more efficiently radiative acceleration by UV photons. However just as G06 pointed out that they had only four sources at the low $v_{\max }$, and their sample of BAL QSOs is biased towards strongly absorbed sources comparing to the BI distribution of SDSS EDR BAL QSOs (Reichard et al. 2003). Giving the importance of this question, more study based on a uniform sample is clearly required.

X-ray absorption is not the only factor that affects the ionization equilibrium of BAL gas. Steffen et al. (2006) showed that the X-ray luminosity of non-BAL QSOs has a large scatter for a given optical luminosity. According to the current popular scenario that BAL and non-BAL are only a matter of whether our line of sight passes through BAL region or not, the intrinsic spectral energy distribution (SED) between UV and X-ray of BAL QSOs should be also diverse. Therefore, it would be interesting to study how the wind properties depend on the intrinsic SED because ionization equilibrium is also closely related to the intrinsic SED. If such a relation does exist, it may offer insight into the driver of the outflows.

In this paper, we present a study of BAL QSOs from SDSS Data Release 5 (DR5) (Adelman-McCarthy et al. 2008) that observed by XMM-Newton satellite in X-ray in order to explore the relations between UV and X-ray absorbers as well as the relations between BAL properties and the intrinsic UV to X-ray spectra. In $\S 2$ we describe the selection of our C IV BAL QSOs sample and the data analysis in $§ 3$. We show our results and discuss the underlying physics in $\S 4$. Finally, we summarize our results in $\S 5$. Throughout the paper, we assume the cosmological parameters $H_{0}=70 \mathrm{~km} \mathrm{~s}^{-1} \mathrm{Mpc}^{-1}, \Omega_{\mathrm{M}}=0.3$ and $\Omega_{\Lambda}=0.7$.

\section{THE BAL QUASARS OBSERVED BY XMM-Newton}

Starting from the spectroscopic quasar sample in the SDSS DR5 Adelman-McCarthy et al. 2008), we compiled a sample of definitive C IV BAL quasars that have been observed by $X M M-N e w t o n$ either serendipitously or as a target. We restricted the redshift $1.5<z<4.0$ in order to make sure that the C IV $\lambda 1549$ is shifted into the SDSS wavelength regime $(3800-9200 \AA)$. We matched these quasars with $X M M$-Newton pointing, and resulted in 225 quasars in the FOV of XMM-Newton observation to date of April,2007.

We measured the BALnicity Index (BI, Weymann et al. 1991) and the maximum outflow velocity for these 225 quasars using our own fitting code (see $§ 3.2$ for detail). We adopted the conventional definition for the BAL QSO: the equivalent width (in $\mathrm{kms}^{-1}$ ) of any contiguous absorption (at least $10 \%$ below the continuum) exceeds $2000 \mathrm{~km} \mathrm{~s}^{-1}$ that falls between 3000-25000 $\mathrm{km} \mathrm{s}^{-1}$ blueshifted from the systematic redshift (Weymann et al. 1991). All quasars with non-zero BI were checked by eye, and ambiguous sources were removed. 
Our final sample consists of $41 \mathrm{C}$ IV BAL quasars, including 5 LoBAL quasars, 22 HiBAL quasars, and 14 BAL quasars with unknown BAL subtype because Mg II is not within the SDSS spctral coverage. Most (25) of them have been included in the large BAL QSOs catalog from SDSS DR3(Trump et al. 2006). In comparison with G06, BAL quasars in our sample cover somewhat larger ranges of redshift $(1.579-3.776)$ and UV luminosity $\left(\log \left(l_{2500}\right): 30.212-32.230\right)$, and are fainter on average (31.055). Our sample has more uniform distributions in BI $\left(3-4610 \mathrm{~km} \mathrm{~s}^{-1}\right.$ with an average $\left.1101 \mathrm{~km} \mathrm{~s}^{-1}\right)$ and in $v_{\max }(5306-25000$ $\mathrm{km} \mathrm{s}^{-1}$ with an average $14482 \mathrm{kms}^{-1}$ ) while G06's sample consists mainly of BAL QSOs with the large BI (with an average $3437 \mathrm{~km} \mathrm{~s}^{-1}$ ).

We notice that there are only four XMM-Newton targeted objects: SDSS J091127.61+055054.1, SDSS J111816.95+074558.1, SDSS J152553.89+513649.1 and SDSS J154359.44+535903.2 (Table 1). The first two are the lensed BAL QSOs (Bade et al. 1997), which will be excluded from the following correlation analysis. The third quasar was observed because of its high optical polarization (Shemmer et al. 2005). The fourth object was observed because of its X-ray detection by previous missions, thus may bias towards X-ray bright sources (Grupe et al. 2003).

Some radio loud BAL QSOs show anomalous X-ray properties in comparison with radio quiet counterparts (Brotherton et al. 2006; Wang et al. 2008). In order to mark such sources, we calculate the radio-to-optical flux ratios, $R_{i}=\log \left(S_{1.4 G H z} / S_{i}\right)$, following the definition of Ivezić et al. (2002). The flux densities at $1.4 \mathrm{GHz}, S_{1.4 G H z}$, are taken from the Faint Images of the Radio Sky at Twenty centimeters survey (FIRST; White et al. 1997). We estimate the $R_{i}$ upper limits by taking the $2 \sigma$ errors as the upper limit of radio flux density. Only, three sources (SDSS J092345.19+512710.0,SDSS J133004.72+472301.0 and SDSS $\mathrm{J} 133553.61+514744.1$ ) have radio counterparts with the measured flux density of $1.72 \mathrm{mJy}$ ,1.18 mJy and $2.92 \mathrm{mJy}$, which give $R_{i}=1.42,1.18$ and 1.14 , respectively.

We listed our sample in Table 1 including the SDSS ID, the redshift, $i$ band fiber magnitude of SDSS, the flux density at rest-frame $2500 \AA\left(f_{2500}\right)$, Galactic $N_{\mathrm{H}}$ from Dickey \& Lockman (1990), the BAL subtype and the radio-to-optical flux ratios $R_{i}$. Also we listed the BI and $v_{\max }$ in Table 1 (see $\S 3.2$ ). The values of $f_{2500}$ are calculated either by averaging the flux densities in the rest-frame range of $2500 \pm 20 \AA$ or by extrapolating from the continuum given by our fitting code. 


\section{DATA ANALYSIS}

\subsection{X-ray Data Analysis}

The X-ray data were retrieved from $X M M$-Newton Science Archive (XSA) and prepared using $S A S 7.0 .0$ with the most recent calibration files. We extracted the background lightcurve above $10 \mathrm{keV}$, and the light curve was used to filter the data obtained during the flaring background periods using a threshold of 1.0 count $\mathrm{s}^{-1}$ for PN and 0.5 count s $\mathrm{s}^{-1}$ for MOS. A sliding box cell detection algorithm (eboxdetect) was applied to the images obtained by PN-CCD detector and two MOS-CCD detectors in the soft $(0.3-2.0 \mathrm{keV})$, hard $(2.0-10.0 \mathrm{keV})$ and full $(0.3-10.0 \mathrm{keV})$ bands to search for X-ray sources. We selected $L=-\ln (P)=10$ as the minimum detection likelihood value, which in turn corresponded to a probability of Poissonian random fluctuations of the counts of $P=4.5 \times 10^{-5}$. Among 41 BAL quasars, 26 were detected in the full band and 25 (13) were detected in the soft (hard) bands at least on one EPIC instrument. The spectra were accumulated from a circle region with a 30 ". radius except for two sources locating close to the edge of the CCD whereas a circle with 20 ". radius was adopted. The backgrounds were extracted from a source-free annulus surrounding each target on the two MOS-CCD detectors and from a source-free circle along the read-out direction on the pn-CCD detector. The photon counts were extracted from the circular source regions centered on the SDSS optical positions with the mentioned radius and the aperture corrections were performed (See Table 2). For non-detections the upper limits of counts are the $90 \%$ confidence limits from Bayesian statistics Kraft et al. 1991). The redistribution matrix file $(r m f)$ and auxiliary response file (arf) were generated using the tasks rmfgen and arfgen respectively.

X-ray spectral modelling are performed using the package XSPEC (Arnaud 1996). We fit all the spectra with a uniform model, an absorbed power law. It is found that the photon index $\Gamma$ of the power-law is around 2.0 with a small scatter for radio-quiet quasars (George et al. 2000; Reeves \& Turner 2000). The broad band X-ray spectra of BAL QSOs are quite similar to those of radio quiet non-BAL QSOs, (Gallagher et al. 2002; Chartas et al. 2002, 2003; Aldcroft \& Green 2003; Grupe et al. 2003; Page et al. 2005), therefore, in following analysis, $\Gamma$ is fixed to 2.0. Both the Galactic neutral HI absorption and an intrinsic absorption are included in the model. The Galactic neutral HI column density is fixed at the value derived from Galactic HI maps(Dickey \& Lockman 1990) (See Table 1). Due to limited count rates and the large uncertainties, we do not consider more complex X-ray absorption models (e.g., a partially covering or ionized absorber), and just adopt a simple neutral absorption with a solar chemical composition at the source rest frame (zwabs in $X S P E C$ ) (Morrison \& McCammon 1983) for the intrinsic absorption. 
To deal with very different X-ray counts available, two different methods are used to estimate the intrinsic absorption column density. The X-ray spectrum is fitted directly with an absorbed power-law if the source is detected in the both soft and hard band. Only the intrinsic absorption and the power-law normalization are free parameters. Other parameters, including redshift, the Galactic neutral absorption and the photon index $\Gamma$, are fixed to the proper values. Either $\chi^{2}$-statistic or C-statistic (Cash 1979) is taken as the merit of the fit depending on the net source counts. If the net source counts are greater than 100, the spectrum is re-binned with at least 15 counts per bin, and the fit is performed by minimizing $\chi^{2}$. Otherwise, the spectrum is not binned and the fit is performed by minimizing the Cstatistics. In order to test the validity of the fixed $\Gamma$ power-law model, we make $\Gamma$ free to fit eight sources with greater than 100 counts. The average value of $\Gamma$ of six sources is about 1.90, which is very close to 2.0. The other two (SDSS J091127.61+055054.1 and SDSS J100728.69+534326.7) show a rather flat spectra with $\Gamma \sim 1.2$. Page et al. (2005) mentioned that SDSS J091127.61+055054.1 should be better modelled by a broken power law with the values of $\Gamma 0.92$ and 1.96,respectively. The fitted intrinsic absorptions are very similar to those listed in Table 3 except the two flat spectral sources. A flat spectrum can be caused by complex absorption, strong reflection component or an intrinsic flat power-law. Because all other six sources show normal X-ray spectra and there is no evidence for strong Fe $\mathrm{K} \alpha$ in the $\mathrm{X}$-ray spectrum, we believe that the flat spectra in these two objects are caused by complex absorptions. However, due to limit counts available, we will not try more complicated models.

For those sources detected only in hard or soft band, the upper/lower limit of the column density of intrinsic absorption is estimated from the hardness ratios, defined as $\mathrm{HR}=(h-s) /(h+s)$, where $h$ and $s$ are referred to the hard and soft band counts, respectively. First for each source, we calculate hardness ratios for a grid absorbed power-law models with column densities in the range of $10^{20}-10^{24} \mathrm{~cm}^{-2}$ using the arf and $\mathrm{rmf}$ at the source position. The observed hardness ratio is then compared to the models and then the upper/lower limit of the intrinsic absorption could be derived.

In order to compare our sample with that of G06, we also calculate $\alpha_{\text {ox }}, \Delta \alpha_{\text {ox }}$ and $\alpha_{\text {ox }}($ corr $), \Delta \alpha_{\text {ox }}($ corr $)$, defined in the G06 11, as follows. First, the above hardness ratios for all BAL QSOs in the sample were estimated. Hardness ratios for a grid of power-law models only absorbed by the Galactic column density in that direction were then calculated using the XSPEC. The observed HR was compared to the model HRs to estimate the photon index $\Gamma_{\mathrm{HR}}$. With the best fitted photon-index, the normalization at $1 \mathrm{keV}$ was derived from the count-rates. The Galactic absorption corrected $2 \mathrm{keV}$ flux is determined from the

\footnotetext{
${ }^{1}$ Our definition of the $\operatorname{soft}(s: 0.3-2.0 \mathrm{keV})$ and hard $(h: 2.0-10.0 \mathrm{keV})$ bands is slightly different from theirs $(s: 0.5-2.0 \mathrm{keV} ; h: 2.0-8.0 \mathrm{keV})$.
} 
model. We defined the UV to X-ray broad band spectral index as $\alpha_{\mathrm{ox}}=0.384 \log \left(f_{2 \mathrm{keV}} / f_{2500}\right)$ (Tananbaum et al. 1979). It is found that $\alpha_{\mathrm{ox}}$ is correlated with the optical luminosity of quasars (Yuan et al. 1998; Avni \& Tananbaum 1986; Wilkes et al. 1994; Green et al. 1995), though its reality has been questioned by Yuan et al. (1998); Tang et al. (2007). Following G06, we introduce a quantity of $\Delta \alpha_{\text {ox }}=\alpha_{\text {ox }}-\alpha_{\text {ox }}\left(l_{2500}\right)$ to characterize the weakness of the X-ray emission of the quasar relative to the average quasars at that UV luminosity, where $\alpha_{\text {ox }}\left(l_{2500}\right)$ was the expected $\alpha_{\text {ox }}$ based on the $2500 \AA$ monochromatic luminosity, $l_{2500}$ (Strateva et al. 2005). For sources not detected in both band, an upper limit to the X-ray flux was derived by assuming $\Gamma=1.0$. Finally, $\alpha_{\text {ox }}$ (corr) and $\Delta \alpha_{\text {ox }}($ corr $)$ were calculated from an "absorption-corrected" value for the $2 \mathrm{keV}$ flux density estimated by a fixed $\Gamma=2.0$ powerlaw model normalized by the counts rate in the observed-frame $2-10 \mathrm{keV}$ bandpass. Note that for two lensed sources, SDSS J091127.61+055054.1 and SDSS J111816.95+074558.1, we do not calculate $\Delta \alpha_{\text {ox }}$ or $\Delta \alpha_{\text {ox }}($ corr $)$ since their intrinsic $l_{2500}$ are unknown.

\subsection{Ultraviolet Spectral Analysis And C IV Absorption-Line Parameters}

Following the procedures described in Zhou et al. (2006), we calculate both the BI of $\mathrm{C}$ IV and Mg II absorption lines and their maximum outflow velocity, $v_{\max }$. Briefly, we use the SDSS composite quasar spectrum Vanden Berk et al. (2001) as the template for continuum and emission line spectrum. The template is reddened and scaled to match the observed quasar spectrum in the absorption line free windows. The BI of C IV and Mg II are calculated following the definition given by Weymann et al. (1991) and Reichard et al. (2003), respectively, as follows,

$$
B I=\int_{0 \text { or } 3000}^{25000} d v\left[1-\frac{F^{o b s}(v)}{0.9 F^{f i t}(v)}\right] C(v)
$$

where $F^{o b s}(v)$ and $F^{f i t}(v)$ are the observed and fitted fluxes, respectively, as a function of velocity in $\mathrm{km} \mathrm{s}^{-1}$ from the systematic redshift within the range of each absorption trough and

$$
C(v)= \begin{cases}1.0, & \text { if }\left[1-\frac{F^{o b s}(v)}{0.9 F^{f i t}(v)}\right]>0 \text { over a continuous interval of } \gtrsim W \mathrm{~km} \mathrm{~s}^{-1} \\ 0, & \text { otherwise }\end{cases}
$$

The integral in equation (1) starts from $v=3000 \mathrm{~km} \mathrm{~s}^{-1}$ for C IV and from $v=0 \mathrm{~km} \mathrm{~s}^{-1}$ for Mg II. The threshold interval in equation (2) is $W=2000 \mathrm{~km} \mathrm{~s}^{-1}$ for C IV and from $W=1000 \mathrm{~km} \mathrm{~s}^{-1}$ for $\mathrm{Mg}$ II. Five sources show the non-zero BI of $\mathrm{Mg}$ II consistent with $\sim 10 \%$ fraction of low ionization BAL QSOs. The maximum outflow velocity are calculated, simultaneously. The BI and $v_{\max }$ of $\mathrm{C}$ IV absorption lines are listed in Table 1. 
Finally, we also remeasure the BI and $v_{\max }$ for G06's sample on the LBQS spectra (Foltz et al. 1987, 1989; Hewett et al. 1991; Chaffee et al. 1991; Morris et al. 1991; Hewett et al. 1995) using our method because we will combine G06's sample with ours in the statistical analysis between the properties of X-ray and UV. We find that our measurements of either $\mathrm{BI}$ or $v_{\max }$ are well correlated with those of G06 although there is considerable scatter. For six objects both in this sample, the SDSS BAL QSO sample of Trump et al. (2006) and in G06, our measurements appear in between theirs. Note that the results of correlation analysis by using G06's BI and $v_{\max }$ are very similar to those obtained by using ours.

\section{RESULTS AND DISCUSSION}

\subsection{X-ray Properties Of BAL QSOs}

To investigate the general X-ray properties of our SDSS/XMM-Newton BAL sample (this paper), we try to measure the intrinsic absorption adopting a simple neutral absorption. However, only 12 of 41 sources can be fitted directly to give the intrinsic absorption column densities $N_{\mathrm{H}}$ in the range $\sim 4 \times 10^{21}$ to $\sim 2 \times 10^{23} \mathrm{~cm}^{-2}$. For 14 sources, upper/lower limits can be placed by the hardness ratios HR. The final sample spans a wide range of intrinsic absorption column densities from $<10^{20} \mathrm{~cm}^{-2}$ to $\sim 10^{24} \mathrm{~cm}^{-2}$ (Table 3). The lowest limit is obtained for the LoBAL QSO, SDSS J092238.43+512121.2. We have rechecked the optical spectrum, the identification of this quasar as LoBAL might be questionable because of the presence of narrow absorption lines. Notably, five LoBAL QSOs do not show stronger absorption than HiBAL QSOs.

Following G06,we measure $\alpha_{\mathrm{ox}}$ or place upper/lower limits on it, which ranges from -1.36 to -2.26 with an average -1.86 (Table 3). Similar to G06, we show the $\Delta \alpha_{\text {ox }}$, to account for the luminosity dependence of $\alpha_{\text {ox }}$ (Table 3, see also the dot-dashed line in Fig. 1). The average value of $\Delta \alpha_{\text {ox }}$ is -0.25 , suggesting that $2 \mathrm{keV} \mathrm{X}$-ray luminosities (at rest frame) of our SDSS/XMM-Newton BAL sample are roughly three times fainter than the SDSS/ROSAT non-BAL sample(Strateva et al. 2005). And in Table 3 we also present the $\alpha_{\mathrm{ox}}$ (corr) and $\Delta \alpha_{\mathrm{ox}}($ corr $)$ as a surrogate of the intrinsic X-ray properties of BAL QSOs. $\alpha_{\text {ox }}($ corr $)$ is calculated by assuming $\Gamma=2.0$ and using the hard-band counts rate to normalize the X-ray continuum and $\Delta \alpha_{\text {ox }}($ corr $)=\alpha_{\text {ox }}($ corr $)-\alpha_{\text {ox }}\left(l_{2500}\right)$ (see G06 or $\S 3.1$ for the definition). We find $\Delta \alpha_{\text {ox }}$ (corr) in the range from -0.36 to 0.29 with an average value of 0.11, which indicates our SDSS/XMM-Newton BAL sample is slightly X-ray brighter, relative to the average quasars at that UV luminosity, than the SDSS/ROSAT non-BAL sample(Strateva et al. 2005, see Fig. 1). The X-ray brighter of our sample may be due to the relative shallower detection threshold of XMM-Newton relative to Chandra. Comparing the 
$\Delta \alpha_{\text {ox }}($ corr $)$ distribution of the LBQS/Chandra BAL sample(G06) with the SDSS/ROSAT non-BAL sample(Strateva et al. 2005, figure 2 of G06) one can find the LBQS/Chandra BAL sample(G06) is slightly intrinsic X-ray weaker with a median $\Delta \alpha_{\text {ox }}($ corr $)=-0.14$ than the normal QSOs. Alternately, even hard X-rays are absorbed in the LBQS/Chandra BAL sample(G06) so that the simple assumption is broken down (See G06 or $\S 3.1$ for detail). We have carried out simulations to test this effect. Using XSPEC, we simulate the dependence of the $\Delta \alpha_{\mathrm{ox}}(\mathrm{corr})$ on the varying neutral hydrogen column density $N_{\mathrm{H}}$. We assume that $\Delta \alpha_{\text {ox }}($ corr $)$ equals to zero for a single $\Gamma=2$ power-law with $N_{\mathrm{H}}=10^{20} \mathrm{~cm}^{-2}$ at the redshift 2. We find that an absorption column density of $3 \times 10^{23} \mathrm{~cm}^{-2}$ is required in order to account for the mean offset, about -0.14 , of $\Delta \alpha_{\text {ox }}($ corr $)$ of the LBQS/Chandra BAL sample(G06) relative to the SDSS/ROSAT non-BAL sample(Strateva et al. 2005). If this is the main cause, most of X-ray weak sources in the LBQS/Chandra BAL sample(G06) will have a column density at least order of this. Future X-ray observation is certainly needed to assess this.

Either intrinsic X-ray weak or large column density of absorber may indicate that the LBQS / Chandra BAL sample(G06) is biased in X-ray properties. Their sample obviously has larger values of BI and $v_{\max }$ than the SDSS BAL QSOs(Reichard et al. 2003) and is not uniform on UV properties too. Note that our SDSS / XMM-Newton BAL sample is more uniform, especially on UV properties, and can be used as a complement to the LBQS/Chandra BAL sample(G06). For direct comparison, we show the distribution of $\Delta \alpha_{\text {ox }}$ for the SDSS/ROSAT non-BAL sample(Strateva et al. 2005) and $\Delta \alpha_{\text {ox }}($ corr $)$ for our SDSS/XMM-Newton BAL sample(this paper) and the LBQS/Chandra BAL QSO sample(G06) in Fig. 1, In the following we used the combined sample of ours 41 and G06's 35 sources to study the relations between X-ray and UV properties. Note again, the UV properties of the LBQS/Chandra BAL QSO sample(G06) used in this paper are obtained by using our procedures so that we can use the consistent definition of BI and $v_{\max }$. We also use the hardness ratios presented in G06 to calculate $N_{\mathrm{H}}$ of these G06 QSOs following the same approach as we have done for XMM-Newton sources.

\subsection{X-ray And UV Absorptions}

One of the purposes of this paper is to study the relationship between the UV and X-ray absorbers. It is generally believed that the X-ray absorber shields the disk winds from soft $\mathrm{X}$-rays and makes line driving more efficient. A naive deduction is that the properties of UV and X-ray absorptions are correlated. Basing on this idea, G06 presented a correlation analysis between the X-ray absorption using $\Delta \alpha_{\mathrm{ox}}$ as an indicator and the UV absorption 
properties such as BI, DI, $v_{\max }$ and $f_{\text {deep }}$. They found only a weak correlation between $\Delta \alpha_{\text {ox }}$ and $v_{\max }$. We will carry out a similar analysis using a larger sample covering more uniformly the whole BI range. In the following analysis, we will use Kendall- $\tau$ test to quantify the significance of a correlation.

First, we check whether $\Delta \alpha_{\text {ox }}$ is a good indicator of X-ray absorption. In the left panel of the Fig. 2, we show $N_{\mathrm{H}}$ versus $\Delta \alpha_{\text {ox }}$ for the combined subsample of 51 BAL QSOs that $N_{\mathrm{H}}$ is obtained either from spectral fit or from HR analysis. Similar to G06, we find a clear correlation between the two quantities. The probability for null hypothesis is less than $0.01 \%$ using non-parametric Kendall $\tau$-test (See Table 4). Then we compare the redshift distributions of sources with $\Delta \alpha_{\mathrm{ox}}>-0.2$ and $\Delta \alpha_{\mathrm{ox}}<-0.2$. The result is their distributions are very similar, which indicates that the correlation between $N_{\mathrm{H}}$ and $\Delta \alpha_{\text {ox }}$ is not from selection effect of redshift. These suggest that $\Delta \alpha_{\text {ox }}$ can be used as a measure for X-ray absorption indeed.

Next, we examine the correlations between BAL properties and the X-ray absorption column density $N_{\mathrm{H}}$ (Fig. 4) measured through X-ray spectral fit or HR analysis. We do not find any correlation with a probability of null hypothesis less than $1 \%$ (Table 4). However, a weak correlation between $\mathrm{BI}$ and $N_{\mathrm{H}}$ cannot be rejected because the large uncertainty in the $N_{\mathrm{H}}$ measurement may reduce the significance of a weak correlation to the measured level $(2 \%)$.

We show $\Delta \alpha_{\text {ox }}$ versus BI and $\Delta \alpha_{\text {ox }}$ versus $v_{\text {max }}$ in Fig. 3. Two lensed BAL QSOs are excluded from following analysis because their UV and X-ray light may have been differntly amplified. There appears a correlation between $\Delta \alpha_{\text {ox }}$ and BI with the probability for null hypothesis of only $0.05 \%$ (Table 4 ). The correlation appears not linear, rather there is an upper envelope. Since LoBAL QSOs may be different from the HiBAL QSOs (Boroson \& Meyers 1992; Wang et al. 2007), we also make Kendall test for 45 HiBAL QSOs only. The correlation is marginally significant with a null probability of $1 \%$. The decrease in significance is caused by reducing the sample size. However, we do not find any significant correlation between $v_{\max }$ and $\Delta \alpha_{\mathrm{ox}}$, which was seen in G06, in neither the whole sample nor in the HiBAL subsample with a null probability of $5 \%$ and $68 \%$, respectively (Table 4). Comparison with the LBQS/Chandra BAL sample(G06), our sample has a handful BAL QSOs on the upper right of the figure. These BAL QSOs destroy the weak correlation trend of $v_{\max }$ vs $\Delta \alpha_{\text {ox }}$ in the LBQS/Chandra BAL sample(G06). These correlations are more or less similar to the correlations using $N_{\mathrm{H}}$ with an exception of higher significance. It is worthwhile to note that $\Delta \alpha_{\text {ox }}$ reflects a combination of the X-ray absorption and the intrinsic deviation to the average quasar SED. Therefore, one must be careful as using it as an indicator of X-ray absorption. We will discuss below the implication of these results. 


\subsection{Intrinsic X-ray Properties And The Outflow}

Previous studies have shown that UV properties, such as the blueshift and the equivalent width of C IV emission line, are correlated with X-ray to optical flux ratio for non-BAL QSOs (e.g. Baskin \& Laor (2004); Richards (2006)). It would be interesting to explore whether the BAL properties are correlated with the intrinsic $\alpha_{\mathrm{ox}}$. Unlike the correlation with X-ray absorption, such correlation should give information for the primary driver of the outflow. Here we use a corrected $\alpha_{\text {ox }}$ i.e. $\alpha_{\text {ox }}$ (corr) to represent the intrinsic $\alpha_{\text {ox }}$ and investigate its relation with the UV absorption line properties. We also study the correlations between UV properties and $\Delta \alpha_{\text {ox }}($ corr $)$ so that we can compare the results with those in previous section and G06. We note that the range of UV luminosity, $l_{2500}$, for the combined sample is only 2 dex, which introduce a scatter in $\alpha_{\text {ox }}$ (corr) through $\alpha_{\text {ox }} \sim l_{2500}$ correlation, of less than 0.27 , a factor of about 2.5 smaller than the dynamic range of $\alpha_{\text {ox }}$ (corr). Therefore, the difference between $\Delta \alpha_{\text {ox }}($ corr $)$ and $\alpha_{\text {ox }}$ (corr) should be small in correlation analysis. This is verified below that the relationships between $\Delta \alpha_{\text {ox }}($ corr $)$ and $\mathrm{UV}$ properties have a very similar behavior as those between $\alpha_{\text {ox }}($ corr $)$ and UV properties.

Before exploring UV and X-ray connection, we first check whether $\alpha_{\text {ox }}($ corr $)$ and $\Delta \alpha_{\text {ox }}($ corr $)$ are affected by the X-ray absorption or not. We plot $N_{\mathrm{H}}$ versus $\Delta \alpha_{\text {ox }}($ corr $)$ on the right panel of Fig. 2. There is no apparent correlation between the two quantities. Kendall test gives a probability of chance coincidence of $36 \%$ (Table 4). Therefore, we can conclude that there is no evidence that $\alpha_{\mathrm{ox}}(\mathrm{corr})$ and $\Delta \alpha_{\mathrm{ox}}(\operatorname{corr})$ are significantly affected by X-ray absorption.

We then explore the correlations between $\alpha_{\text {ox }}$ (corr) and BI or $v_{\max }$ with Kendall and Spearman tests. We find that $\alpha_{\text {ox }}$ (corr) is significantly correlated with both BI and $v_{\max }$ with a Null probability of less than $0.1 \%$ for either test(See Fig 5; also Table 4). The correlation is still very significant $(P<0.1 \%)$ for HiBAL QSO subsample (45 QSOs). For clarity we show only QSOs detected in the hard X-ray band in Fig,6. Furthermore, these QSOs are more important for the Kendall and Spearman tests than the rest objects, and can give us a clear trend about these correlations. We note that one LoBAL QSOs, SDSS J133553.61+514744.1, which appears largely discrepant with the main sample in Fig. 5 and 6. This quasar has very steep X-ray photon index $\Gamma \sim 2.56$ so that our 'absorptioncorrection' underestimate the intrinsic X-ray luminosity. If we set $\alpha_{\mathrm{ox}}=-1.82$ as the lower limit of $\alpha_{\text {ox }}(\operatorname{corr})$ (it is reasonable since $\alpha_{\text {ox }}$ (corr) is larger than $\alpha_{\text {ox }}$ ), this quasar would move rightward and is consistent with other quasars. The correlations between $\Delta \alpha_{\text {ox }}($ corr $)$ and UV properties are very similar to above correlations using $\alpha_{\text {ox }}$ (corr) except the latter appear slightly more significant (Table 4). This may indicate that the dependence of UV properties on $\alpha_{\text {ox }}($ corr $)$ is more fundamental than on $\Delta \alpha_{\text {ox }}($ corr $)$.

Is it possible that these correlations are introduced by some selection effect in the sam- 
ple? If it is the case this effect would tend to miss the objects which occupy the bottom-left (X-ray weak and high UV absorption) and top-right (X-ray strong and low UV absorption) corners of Fig. 5 and 6. Note that the sample selections of ours and G06's are both based on the optical luminosity and have nothing to do with the X-ray properties. If the relative X-ray luminosity is uncorrelated with BAL properties it is hard to understand why G06 and we select the objects at the bottom-right(top-left) corner but miss those at the bottom-left(topright) corner. Since objects at top-right (bottom-left) corners, if they really exist, should have the same optical properties as these at top-left (bottom-right). All of these analysis indicate the correlations between the intrinsic $\alpha_{\mathrm{ox}}$ and the properties of UV absorber are real. We discuss the implications of the correlations in details in the next subsection.

\subsection{Discussion}

Using a larger and more uniform sample, we reexamine the correlations between BAL properties and X-ray absorption presented in G06. Two indicators of X-ray absorption, $N_{\mathrm{H}}$ and $\Delta \alpha_{\mathrm{ox}}$, are used in the work. We identify the correlation between $\Delta \alpha_{\mathrm{ox}}$ and $B I$ as the only significant one. In particular, we do not find the correlation between $\Delta \alpha_{\text {ox }}$ and $v_{\max }$ claimed in G06, and any correlation between $N_{\mathrm{H}}$ and the UV absorption properties. Although we can not rule out a weak correlation between $N_{\mathrm{H}}$ and the UV absorption line properties due to relative large error bar of $N_{\mathrm{H}}$ our results clearly suggest that X-ray absorption is not the major factor that determines UV absorption properties. Given the fact that almost all BAL QSOs show strong absorption in X-ray, it seems that X-ray absorption is a necessary condition for launching of the BAL winds, but the properties of the wind depend on other factors. As shown above, the observed correlation between $\Delta \alpha_{\text {ox }}$ and BI may be the secondary effect of the correlation between BI and $\alpha_{\text {ox }}$ (corr) or $\Delta \alpha_{\text {ox }}($ corr $)$, as $\Delta \alpha_{\text {ox }}$ is composed of the contributions of absorption and of $\Delta \alpha_{\text {ox }}($ corr $)$.

In passing, we note that lack of correlations between the X-ray absorption column density and UV properties does not necessarily contradict with the scenario of radiatively accelerated wind as naively thought. For locally optically thin material, the ratio of the radiation force to the gravitational force is a function of Eddington ratio and the cross-section ratio of effective absorption to Thomson scattering. If resonant scattering is responsible for the absorption opacity, the cross-section will be determined by the fraction Li-like ions. According to the equatorial wind model(Murray et al. 1995), a clump of highly ionized gas (shielding gas), which accounts for most X-ray opacity, blocks the soft X-ray interior to the wind. The transmitted flux of soft X-rays that ionize Li-like ions in the wind depends strongly on the X-ray column density, $N_{\mathrm{H}}$. If $N_{\mathrm{H}}$ along the direction is very small, high- 
velocity wind cannot be launched because of the reduction of the radiation force caused by the over-ionization. On the other hand, if $N_{\mathrm{H}}$ is very large, the wind will end up with a turbulent flow due to the blocking of thick very-low-ionized gas behind(see the figure 4 of Proga et al. 2000). Thus, high-velocity wind can only be launched when the radial column density $N_{\mathrm{H}}$ is moderate as the fraction of Li-like ions, such as $\mathrm{C}$ IV and N V, is large enough. As far as the X-ray absorption column density is in the right range, the fraction of Li-like ions should be the dominant species. The flow properties are then determined self-consistently by the launching radius, the gas density at the launching radius and the radiation intensity. If X-ray absorber is well separated from the UV absorber, then we would not expect any correlation between the X-ray absorption column density and the flow properties for BAL QSOs apart.

On the other hand, the X-ray absorber may be the 'hitchhiking' gas just located at the inner edge of the wind, and its properties may have a close connection with the boundary conditions of the disk wind(Murray et al. 1995). In that case, we should consider globally the structure of gas along a line of sight. The wind starts at a radius where the radiation force is substantially larger than the gravitational force. As far as the gas density is high enough, such a region can certainly exist. Murray et al. (1995) has worked out a consistent line acceleration model, and they found that gas column density and final velocity are correlated for a constant Eddington ratio and at a given launch radius. However, if the launching radius is not exactly scaled with luminosity as $L^{1 / 2}$ and there are a range of Eddington ratio, as they assumed, the correlation can be smeared out.

More interesting results of our work are the strong correlations between the parameters of outflow and intrinsic $\alpha_{\text {ox }}$. We argue that these correlations are essential rather than due to some selection effect or the secondary effect of other correlations (see previous subsection for details). In fact our results are consistent with Richards (2006) who found that the QSOs with large blueshifts of C IV emission line, i.e. the parent population of BAL QSOs as suggested by Richards (2006), tend to have lower X-ray luminosity for given optical luminosity (their figure 5). It is also upheld by Laor \& Brandt (2002) who presented significant correlation between the equivalent width of $\mathrm{C}$ IV absorption and $\alpha_{\mathrm{ox}}$ in a sample of non-BAL QSOs. This correlation is actually predicted by Murray et al. (1995), in which they found that quasars with a large X-ray to UV ratio can only produce weak low velocity winds while quasars with a small X-ray to UV ratio can produce strong and large velocity winds. This is exactly what we have found here. As we discussed above, their model also predicted a correlation between the X-ray absorption column density and the maximum velocity of the flow, which is not observed in this sample. Lack of such correlation may be due to two important factors that (1) variation in the Eddington ratio and launching radius; (2) the large uncertainties in the measurement of absorption column density. 
We do not fully understand why the variation in the Eddington ratio and launching does not completely smeared out the correlation with $\alpha_{\text {ox }}$ (corr). There seems one reason for this. Wang et al. (2004) found that the 2-10kev luminosities to bolometric luminosities ratio tightly anti-correlated with Eddington ratio for a sample of broad-line and narrow-line Seyfert 1 AGNs. If this correlation holds up for BAL QSOs, one would expect that quasars with high Eddington ratio would have larger radiative acceleration force, or large terminal velocity, and at the same time X-ray weaker. Ganguly et al. (2007) find that $v_{\max }$ as a function of Eddington ratio has an upper envelope in the SDSS3 BAL catalog, exactly as expected. Since there is no clear correlation of BI with UV luminosities(cf. Laor \& Brandt 2002), Eddington rate and black hole mass(Ganguly et al. 2007), it is very likely that the BAL properties are more likely determined by the SED of quasars rather than Eddington ratio.

\section{SUMMARY}

We compile a large C IV BAL QSOs sample from the XMM-Newton archive data and SDSS DR5. The sample consists of 41 BAL QSOs, among which 26 QSOs are detected in the $\mathrm{X}$-ray band. Our sample spans wide and homogeneous ranges of both BI and $v_{\max }$ and can be used to complement the LBQS/Chandra BAL sample(G06). In addition, the combined sample of ours and G06s show a more homogeneous distribution of intrinsic X-ray properties than G06s. Using this combined sample, we investigate the correlations between X-ray and UV properties of BAL QSOs.

We briefly summarize our conclusions below:

1. We confirm the previous results that BAL QSOs are generally soft X-ray weak, which is mainly due to the intrinsic X-ray absorption. We also find the X-ray luminosities of BAL QSOs with given optical luminosity have large scatter. The scatter is caused by both the various column densities of X-ray absorber and the scatter of intrinsic X-ray emission at given optical luminosity.

2. We do not find any evidence for the claimed correlation between the BAL properties and soft X-ray absorption, with an exception of the correlation between BI and $\Delta \alpha_{\text {ox }}$. The correlation between BI and $\Delta \alpha_{\text {ox }}$ can be induced by the correlation between BI and the intrinsic $\alpha_{\mathrm{ox}}$. The X-ray absorber is important for launching the high-velocity wind but do not directly determine the BAL properties.

3. There are significant correlations between intrinsic X-ray strength, $\alpha_{\mathrm{ox}}(\mathrm{corr})$, and BI and $v_{\max }$ in the combined sample. These correlations are essential rather than due 
to any artificiality. We preliminarily interpret that the BAL properties are influenced by the intrinsic SED of QSOs, which is consistent with the prediction of a radiatively accelerated disk wind model (Murray et al. 1995).

We thank the anonymous referee for helpful comments. We thank Paul Hewett for providing electronic data of LBQS spectra.We also acknowledge the Sloan Digital Sky Survey(http://www.sdss.org This work was supported by the Knowledge Innovation Program of the Chinese Academy of Sciences, Grant No. KJCX2-YW-T05 and the National Basic Research Program of China (973 Program) under Grant No. 2007CB815400.

\section{REFERENCES}

Adelman-McCarthy, J. K., et al. 2008, ApJS, 175, 297

Aldcroft, T. L. \& Green, P. J., 2003,ApJ,592,710

Arnaud, K. A., 1996, in ASP Conf. Ser. 101: Astronomical Data Analysis Software and Systems V,eds. G. Jacoby \& J. Barnes vol. 5,17

Avni, Y., \& Tananbaum, H. 1986, ApJ, 305, 83

Bade, N., Siebert, J., Lopez, S., Voges, W., \& Reimers, D. 1997, A\&A, 317, L13

Baskin, A., \& Laor, A. 2004, MNRAS, 350, L31

Boroson, T. A., \& Meyers, K. A. 1992, ApJ, 397, 442

Brandt, W. N., Laor, A., \& Wills, B. J. 2000, ApJ, 528, 637

Brinkmann, W., Wang, T., Matsuoka, M., \& Yuan, W. 1999, A\&A, 345, 43

Brotherton, M. S., De Breuck, C., \& Schaefer, J. J. 2006, MNRAS, 372, L58

Cash,W., 1979, ApJ, 228,939

Chaffee, F. H., Foltz, C. B., Hewett, P. C., Francis, P. A., Weymann, R. J., Morris, S. L., Anderson, S. F., \& MacAlpine, G. M. 1991, AJ, 102, 461

Chartas, G.,Brandt, W. N. \& Gallagher, S. C., 2003, ApJ, 595,85

Chartas, G.,Brandt, W. N.,Gallagher, S. C. \& Garmire, G. P., 2002, ApJ, 579 , 169 
Dickey,J. M. \& Lockman, F. J., 1990, ARA\&A, 28,385

Foltz, C. B., Chaffee, F. H., Jr., Hewett, P. C., MacAlpine, G. M., Turnshek, D. A., Weymann, R. J., \& Anderson, S. F. 1987, AJ, 94, 1423

Foltz, C. B., Chaffee, F. H., Hewett, P. C., Weymann, R. J., Anderson, S. F., \& MacAlpine, G. M. 1989, AJ, 98, 1959

Gallagher, S. C., Brandt, W. N., Sambruna, R. M., Mathur, S., \& Yamasaki, N. 1999, ApJ, 519,549

Gallagher, S. C., Brandt, W. N., Chartas, G. \& Garmire, G. P., 2002, ApJ, 567 , 37

Gallagher, S. C., Brandt, W. N., Chartas, G., Priddey, R., Garmire, G. P., \& Sambruna, R. M. 2006, ApJ, 644, 709

Gallagher, S. C., Brandt, W. N., Wills, B. J., Charlton, J. C., Chartas, G., \& Laor, A. 2004, ApJ, 603, 425

Ganguly, R., Brotherton, M. S., Cales, S., Scoggins, B., Shang, Z., \& Vestergaard, M. 2007, ApJ, 665, 990

Gehrels,N.,1986,ApJ, 303,336

George, I. M., Turner, T. J., Yaqoob, T., Netzer, H., Laor, A., Mushotzky, R. F., Nandra, K., \& Takahashi, Y., 2000, ApJ, 531,52

Green, P. J., et al. 1995, ApJ, 450, 51

Grupe, D., Mathur, S., \& Elvis, M., 2003,AJ,126,1159

Hewett, P. C., \& Foltz, C. B. 2003, AJ, 125, 1784

Hewett, P. C., Foltz, C. B., \& Chaffee, F. H. 1995, AJ, 109, 1498

Hewett, P. C., Foltz, C. B., Chaffee, F. H., Francis, P. J., Weymann, R. J., Morris, S. L., Anderson, S. F., \& MacAlpine, G. M. 1991, AJ, 101, 1121

Ivezić, Ž., et al. 2002, AJ, 124, 2364

Kraft,R.P.,Burrows,D.N.,\& Nousek,J.A.,1991,ApJ,374,344

Laor, A., \& Brandt, W. N. 2002, ApJ, 569, 641 
Morris, S. L., Weymann, R. J., Anderson, S. F., Hewett, P. C., Francis, P. J., Foltz, C. B., Chaffee, F. H., \& MacAlpine, G. M. 1991, AJ, 102, 1627

Morrison, R. \& McCammon, D., 1983, ApJ, 270 ,119

Murray, N., Chiang, J., Grossman, S. A., \& Voit, G. M. 1995, ApJ, 451, 498

Ogle, P. M., Cohen, M. H., Miller, J. S., Tran, H. D., Goodrich, R. W., \& Martel, A. R. 1999, ApJS, 125, 1

Page, K. L., Reeves, J. N.,OB́rien, P. T.,\& Turner, M. J. L.,2005,MNRAS,898

Park, T., Kashyap, V. L., Siemiginowska, A., van Dyk, D. A., Zezas, A., Heinke, C., \& Wargelin, B. J. 2006, ApJ, 652, 610

Proga, D., Stone, J. M., \& Kallman, T. R. 2000, ApJ, 543, 686

Reeves, J. N. \& Turner, M. J. L., 2000,MNRAS, 316,234

Reichard, T. A., et al. 2003, AJ, 125, 1711

Richards, G. T. 2006, ArXiv Astrophysics e-prints, arXiv:astro-ph/0603827

Shemmer, O., Brandt, W. N., Gallagher, S. C., Vignali, C., Boller, T., Chartas, G., \& Comastri, A. 2005, AJ, 130, 2522

Steffen, A. T., Strateva, I., Brandt, W. N., Alexander, D. M., Koekemoer, A. M., Lehmer, B. D., Schneider, D. P., \& Vignali, C. 2006, AJ, 131, 2826

Strateva,I.V.,Brandt, W.N.,Schneider,D.P.,Vanden Berk,D.G.,\& Vignali,C. 2005,AJ,130,387

Tananbaum,H.,et al. 1979,ApJ,234,L9

Tang, S. M., Zhang, S. N., \& Hopkins, P. F. 2007, MNRAS, 377, 1113

Trump, J. R., et al. 2006, ApJS, 165, 1

Vanden Berk, D. E., et al. 2001, AJ, 122, 549

Wang, J., Jiang, P., Zhou, H., Wang, T., Dong, X., \& Wang, H. 2008, ApJ, 676, L97

Wang, J.-M., Watarai, K.-Y., \& Mineshige, S. 2004, ApJ, 607, L107

Wang, T. G., Brinkmann, W., Yuan, W., Wang, J. X., \& Zhou, Y. Y. 2000, ApJ, 545, 77

Wang, T. G., Wang, J. X., Brinkmann, W., \& Matsuoka, M. 1999, ApJ, 519, L35 
Wang, H.-Y., Wang, T.-G., \& Wang, J.-X. 2005, ApJ, 634, 149

Wang, H.-Y., Wang, T.-G., \& Wang, J.-X. 2007, ApJS, 168, 195

Weymann,R.J., Morris,S.L.,Foltz,C.B.,\& Hewett,P,C., 1991,ApJ,373,23

White, R. L., Becker, R. H., Helfand, D. J., \& Gregg, M. D. 1997, ApJ, 475, 479

Wilkes, B. J., Tananbaum, H., Worrall, D. M., Avni, Y., Oey, M. S., \& Flanagan, J. 1994, ApJS, 92, 53

Yuan, W., Siebert, J., \& Brinkmann, W. 1998,A\&A, 334, 498

Zhou, H., Wang, T., Wang, H., Wang, J., Yuan, W., \& Lu, Y. 2006, ApJ, 639, 716 
Table 1. Observed BAL QSOs

\begin{tabular}{|c|c|c|c|c|c|c|c|c|}
\hline Name $(\text { SDSS })^{\mathrm{a}}$ & $z^{b}$ & $\mathrm{i}^{\mathrm{c}}$ & $f_{2500}{ }^{\mathrm{d}}$ & $N_{\mathrm{H}}{ }^{\mathrm{e}}$ & $\mathrm{BI}^{\mathrm{f}}$ & $v_{\max } \mathrm{g}$ & BAL Type ${ }^{\text {h }}$ & $R_{i}{ }^{\mathrm{i}}$ \\
\hline J020230.66-075341.2 & 1.722 & 18.69 & 4.69 & 2.13 & 245 & 6518 & $\mathrm{Hi}$ & $<0.68$ \\
\hline J023224.87-071910.5 & 1.597 & 18.14 & 9.81 & 3.02 & 450 & 7855 & $\mathrm{Hi}$ & $<0.44$ \\
\hline J024304.68+000005.4 & 1.995 & 18.19 & 7.96 & 3.56 & 360 & 5847 & $\mathrm{Hi}$ & $<0.81$ \\
\hline J085551.24+375752.2 & 1.929 & 18.19 & 8.98 & 2.92 & 268 & 20777 & $\mathrm{Hi}$ & $<0.40$ \\
\hline J090928.50+541925.9 & 3.760 & 19.58 & 1.02 & 2.09 & 2890 & 12086 & $\mathrm{H}$ & $<0.97$ \\
\hline J091127.61+055054.1 & 2.793 & 17.77 & 11.38 & 3.64 & 296 & 20368 & $\mathrm{H}$ & $<0.32$ \\
\hline J091400.95+410600.9 & 2.052 & 19.51 & 2.10 & 1.80 & 258 & 18625 & $\mathrm{Hi}$ & $<0.92$ \\
\hline J092138.45+301546.9 & 1.590 & 17.97 & 9.77 & 1.90 & 264 & 12479 & $\mathrm{Hi}$ & $<0.41$ \\
\hline J092238.43+512121.2 & 1.753 & 20.11 & 1.36 & 1.43 & 900 & 10823 & Lo & $<1.14$ \\
\hline J092345.19+512710.0 & 2.168 & 19.14 & 3.18 & 1.42 & 1832 & 18755 & $\mathrm{Hi}$ & 1.42 \\
\hline J092507.54+521102.6 & 2.995 & 19.10 & 4.22 & 1.46 & 2982 & 14990 & $\mathrm{H}$ & $<0.80$ \\
\hline J094309.56+481140.5 & 1.809 & 18.72 & 5.43 & 1.21 & 106 & 22401 & $\mathrm{Hi}$ & $<0.59$ \\
\hline J094440.42+041055.6 & 1.984 & 18.23 & 7.42 & 3.63 & 1934 & 15095 & Lo & $<0.50$ \\
\hline J095110.56+393243.9 & 1.716 & 19.68 & 1.97 & 1.57 & 2328 & 11371 & $\mathrm{Hi}$ & $<1.07$ \\
\hline J100728.69+534326.7 & 1.772 & 19.06 & 3.37 & 0.74 & 3 & 6656 & $\mathrm{Hi}$ & $<0.80$ \\
\hline $\mathrm{J} 105201.35+441419.8$ & 1.791 & 18.47 & 5.06 & 1.12 & 617 & 10192 & $\mathrm{Hi}$ & $<0.55$ \\
\hline J110853.98+522337.9 & 1.665 & 18.53 & 5.81 & 0.90 & 87 & 7266 & $\mathrm{Hi}$ & $<0.55$ \\
\hline $\mathrm{J} 111816.95+074558.1$ & 1.735 & 15.89 & 64.89 & 3.53 & 5 & 5306 & $\mathrm{Hi}$ & $<-0.45$ \\
\hline $\mathrm{J} 112055.78+431412.5$ & 2.389 & 18.66 & 4.22 & 2.07 & 3684 & 20103 & $\mathrm{H}$ & $<0.64$ \\
\hline $\mathrm{J} 112432.14+385104.3$ & 3.530 & 19.99 & 1.75 & 2.06 & 236 & 12438 & $\mathrm{H}$ & $<1.24$ \\
\hline $\mathrm{J} 113419.96+485805.7$ & 3.080 & 20.05 & 2.29 & 1.60 & 1988 & 19192 & $\mathrm{H}$ & $<1.26$ \\
\hline $\mathrm{J} 113406.87+525959.0$ & 1.769 & 18.84 & 4.71 & 1.11 & 1046 & 8581 & $\mathrm{Hi}$ & $<0.67$ \\
\hline $\mathrm{J} 120449.77+020635.6$ & 2.776 & 19.24 & 3.43 & 1.88 & 518 & 19690 & $\mathrm{H}$ & $<0.85$ \\
\hline $\mathrm{J} 120522.18+443140.4$ & 1.921 & 18.42 & 5.62 & 1.27 & 772 & 22284 & $\mathrm{Hi}$ & $<0.57$ \\
\hline $\mathrm{J} 122708.29+012638.4$ & 1.954 & 19.07 & 2.94 & 1.84 & 835 & 19612 & $\mathrm{Hi}$ & $<1.58$ \\
\hline $\mathrm{J} 125741.41+565214.2$ & 1.841 & 19.36 & 2.59 & 1.27 & 1811 & 18178 & $\mathrm{Hi}$ & $<1.00$ \\
\hline $\mathrm{J} 132827.07+581836.9$ & 3.140 & 18.53 & 3.49 & 1.37 & 85 & 6323 & $\mathrm{H}$ & $<0.27$ \\
\hline $\mathrm{J} 133004.72+472301.0$ & 2.825 & 19.16 & 2.38 & 1.55 & 4020 & 17357 & $\mathrm{H}$ & 1.18 \\
\hline $\mathrm{J} 133553.61+514744.1$ & 1.838 & 18.08 & 7.48 & 1.11 & 486 & 9359 & Lo & 1.14 \\
\hline $\mathrm{J} 133639.40+514605.2$ & 2.229 & 19.04 & 2.65 & 1.11 & 2319 & 8631 & $\mathrm{Hi}$ & $<0.80$ \\
\hline J134145.12-003631.0 & 2.215 & 18.52 & 5.86 & 2.06 & 1519 & 8085 & Lo & $<0.58$ \\
\hline J142555.22+373900.7 & 2.731 & 19.13 & 2.69 & 0.94 & 53 & 24676 & $\mathrm{H}$ & $<0.55$ \\
\hline J142539.38+375736.7 & 1.897 & 18.02 & 8.52 & 0.95 & 190 & 24464 & $\mathrm{Hi}$ & $<0.08$ \\
\hline J142652.94+375359.9 & 1.812 & 19.12 & 3.43 & 0.95 & 43 & 20131 & $\mathrm{Hi}$ & $<0.57$ \\
\hline
\end{tabular}


Table 1-Continued

\begin{tabular}{cccrcrrrrc}
\hline \hline Name(SDSS) & a & $z^{\mathrm{b}}$ & $\mathrm{i}^{\mathrm{c}}$ & $f_{2500}{ }^{\mathrm{d}}$ & $N_{\mathrm{H}}{ }^{\mathrm{e}}$ & BI $^{\mathrm{f}}$ & $v_{\max }{ }^{\mathrm{g}}$ & BAL Type $^{\mathrm{h}}$ & $R_{i}{ }^{\mathrm{i}}$ \\
& & & & & & & & \\
\hline $\mathrm{J} 144027.00+032637.9$ & 2.136 & 18.74 & 4.05 & 2.77 & 1141 & 20041 & $\mathrm{Lo}$ & $<0.68$ \\
$\mathrm{~J} 144625.48+025548.6$ & 1.883 & 18.97 & 4.31 & 3.06 & 360 & 6665 & $\mathrm{Hi}$ & $<0.71$ \\
$\mathrm{~J} 150824.22-000603.8$ & 1.578 & 18.44 & 6.73 & 4.58 & 192 & 10208 & $\mathrm{Hi}$ & $<0.51$ \\
$\mathrm{~J} 152553.89+513649.1$ & 2.883 & 16.57 & 29.85 & 1.57 & 754 & 17965 & $\mathrm{H}$ & $<-0.46$ \\
$\mathrm{~J} 153229.97+323658.4$ & 3.048 & 19.22 & 1.94 & 2.03 & 2614 & 14090 & $\mathrm{H}$ & $<0.85$ \\
$\mathrm{~J} 154359.44+535903.2$ & 2.370 & 16.96 & 19.76 & 1.25 & 29 & 13292 & $\mathrm{H}$ & $<-0.11$ \\
$\mathrm{~J} 164151.84+385434.2$ & 3.776 & 18.51 & 7.56 & 1.21 & 4610 & $>25000$ & $\mathrm{H}$ & $<0.57$ \\
\hline
\end{tabular}

${ }^{a}$ SDSS ID.

$\mathrm{b}$ redshift.

${ }^{c_{i}}$ band fiber magnitude of SDSS.

d The rest-frame $2500 \AA$ flux density(in units of $10^{-17} \mathrm{erg} \mathrm{cm}^{-2} \mathrm{~s}^{-1} \AA^{-1}$ )

e The values for $N_{\mathrm{H}}$ (in units of $10^{20} \mathrm{~cm}^{-2}$ ) are from Galactic HI maps (Dickey \& Lockman 1990).

${ }^{\mathrm{f}}$ The BALnicity Index (BI; in units of $\mathrm{km} \mathrm{s}^{-1}$ )

g The maximum outflow velocity $\left(v_{\max }\right.$; in units of $\mathrm{km} \mathrm{s}^{-1}$ )

h The BAL subtype. "Hi" denotes a HiBAL-only object; "Lo" denotes a LoBAL detected through Mg II absorption; "H" denotes a HiBAL object in which the Mg II region is not within the spectral coverage.

${ }^{\text {i } T h e ~ r a d i o-t o-o p t i c a l ~ f l u x ~ r a t i o s, ~} R_{i}=\log \left(S_{1.4 G H z} / S_{i}\right)$, following the definition of Ivezić et al. (2002). 
Table 2. XMM-Newton Observing Log

\begin{tabular}{|c|c|c|c|c|c|c|c|c|}
\hline Name(SDSS) & Obs.ID ${ }^{\mathrm{a}}$ & Date & $T_{\exp }^{\mathrm{b}}$ & Instrument & $\mathrm{Soft}^{\mathrm{c}}$ & $\operatorname{Hard}^{\mathrm{c}}$ & Counts Rate ${ }^{\mathrm{d}}$ & $\mathrm{HR}^{\mathrm{e}}$ \\
\hline J020230.66-075341.2 & 0411980201 & 2006-07-03 & 8.16 & pn & $<7.9$ & $<16.5$ & $<1.81$ & \\
\hline J023224.87-071910.5 & 0200730401 & 2004-01-07 & 37.51 & pn & $64_{-15.8}^{+17.6}$ & $35_{-13.8}^{+15.6}$ & $2.64_{-0.57}^{+0.62}$ & $-0.29_{-0.19}^{+0.17}$ \\
\hline $\mathrm{J} 024304.68+000005.4$ & 0111200101 & $2000-07-29$ & 38.51 & $\operatorname{mos} 1$ & $76_{-17.5}^{+19.8}$ & $54_{-15.2}^{+17.8}$ & $3.39_{-0.61}^{+0.66}$ & $-0.17_{-0.12}^{+0.13}$ \\
\hline $\mathrm{J} 085551.24+375752.2$ & 0302581801 & $2005-10-10$ & 28.45 & $\operatorname{mos} 1$ & $48_{-14.7}^{+17.5}$ & $12_{-11.5}^{+12.2}$ & $2.11_{-0.71}^{+0.61}$ & $\begin{array}{r}-0.12 \\
-0.60_{-0.18}^{+0.05}\end{array}$ \\
\hline J090928.50+541925.9 & 0200960101 & $2005-03-28$ & 71.05 & $\operatorname{mos} 2$ & $<18.0$ & $<22.3$ & $<0.40$ & $\ldots$ \\
\hline J091127.61+055054.1 & $0083240201(\mathrm{~T})$ & $2001-11-02$ & 8.93 & pn & $277_{-34.2}^{+35.9}$ & $151_{-29.2}^{+31.1}$ & $47.94_{-5.10}^{+5.24}$ & $-0.29_{-0.08}^{+0.08}$ \\
\hline J091400.95+410600.9 & 0147671001 & $2003-04-27$ & 13.37 & $\operatorname{mos} 1$ & $<5.8$ & $<8.6$ & $<0.72$ & $\ldots$ \\
\hline J092138.45+301546.9 & 0150620101 & $2003-04-23$ & 15.76 & $\operatorname{mos} 1$ & $11_{-6.8}^{+8.7}$ & $<23.4$ & $1.59_{-0.78}^{+0.79}$ & $<0.35$ \\
\hline J092238.43+512121.2 & 0300910301 & $2005-10-08$ & 15.57 & pn & $173_{-25.7}^{+27.5}$ & $<13.8$ & $10.73_{-1.91}^{+2.08}$ & $<-0.85$ \\
\hline J092345.19+512710.0 & 0300910301 & $2005-10-08$ & 15.55 & pn & $31_{-15.6}^{+17.3}$ & $<9.8$ & $0.32_{-0.31}^{+1.54}$ & $<-0.52$ \\
\hline J092507.54+521102.6 & 0201130501 & $2004-11-15$ & 46.54 & $\operatorname{mos} 1$ & $\begin{array}{l}-15.6 \\
45_{-12.0}^{+13.8}\end{array}$ & $<29.5$ & $1.35_{-0.34}^{+0.31}$ & $<-0.21$ \\
\hline J094309.56+481140.5 & 0201470101 & $2004-10-14$ & 30.35 & $\operatorname{mos} 1$ & $<14.6$ & $<25.2$ & $<0.95$ & $\ldots$ \\
\hline J094440.42+041055.6 & 0201290301 & $2004-05-18$ & 21.27 & $\operatorname{mos} 1$ & $<6.5$ & $<11.1$ & $<0.50$ & $\ldots$ \\
\hline J095110.56+393243.9 & 0111290101 & 2001-11-03 & 21.10 & $\operatorname{mos} 1$ & $<11.1$ & $<11.7$ & $<0.77$ & \\
\hline $\mathrm{J} 100728.69+534326.7$ & 0070340201 & $2001-05-10$ & 19.69 & pn & $135_{-22.3}^{+24.1}$ & $43_{-15.5}^{+17.3}$ & $9.04_{-1.40}^{+1.49}$ & $-0.52_{-0.12}^{+0.11}$ \\
\hline $\mathrm{J} 105201.35+441419.8$ & 0146990901 & $2003-05-24$ & 5.11 & pn & $32_{-10.4}^{+2.2 .3}$ & $25_{-11.2}^{+13.1}$ & $11.16_{-3.06}^{+3.40}$ & $-0.12_{-0.25}^{+0.23}$ \\
\hline $\mathrm{J} 110853.98+522337.9$ & 0304071201 & $2005-10-21$ & 8.18 & $\operatorname{mos} 2$ & $<21.3$ & $<8.1$ & $<3.09$ & $\ldots$ \\
\hline J111816.95+074558.1 & $0203560401(\mathrm{~T})$ & $2004-06-26$ & 68.34 & pn & $8762_{-161.8}^{+162.2}$ & $2598_{-92.1}^{+93.9}$ & $166.24_{-2.69}^{+2.79}$ & $-0.54_{-0.01}^{+0.01}$ \\
\hline $\mathrm{J} 112055.78+431412.5$ & 0107860201 & 2001-05-08 & 21.96 & $\operatorname{mos} 1$ & $<5.8$ & $<13.4$ & $<0.57$ & \\
\hline $\mathrm{J} 112432.14+385104.3$ & 0052140201 & 2001-12-03 & 24.55 & pn & $31_{-13.0}^{+14.8}$ & $<20.4$ & $1.63_{-0.72}^{+0.80}$ & $<-0.21$ \\
\hline J113419.96+485805.7 & 0149900201 & $2003-11-24$ & 14.96 & pn & $<13.6$ & $<17.5$ & $<1.43$ & $\ldots$ \\
\hline $\mathrm{J} 113406.87+525959.0$ & 0200431301 & $2004-11-04$ & 10.82 & $\operatorname{mos} 2$ & $2_{-1.8}^{+6.9}$ & $<10.4$ & $0.52_{-0.51}^{+0.80}$ & $<0.70$ \\
\hline $\mathrm{J} 120449.77+020635.6$ & 0093060101 & $2001-12-21$ & 14.21 & $\operatorname{mos} 1$ & $<11.6$ & $<7.4$ & $<0.99$ & \\
\hline $\mathrm{J} 120522.18+443140.4$ & 0156360101 & $2003-06-11$ & 23.95 & pn & $92_{-20.3}^{+22.2}$ & $47_{-19.2}^{+21.0}$ & $5.80_{-1.18}^{+1.26}$ & $-0.32_{-0.20}^{+0.17}$ \\
\hline $\mathrm{J} 122708.29+012638.4$ & 0110990201 & $2001-06-23$ & 9.56 & pn & $\begin{array}{l}-20.3 \\
22_{-12.2}^{+14.0}\end{array}$ & $\begin{array}{l}-19.2 \\
<12.4\end{array}$ & $2.51_{-1.60}^{-1.18}$ & $<-0.28$ \\
\hline $\mathrm{J} 125741.41+565214.2$ & 0081340201 & 2001-06-07 & 21.39 & $\operatorname{mos} 1$ & $4_{-4.0}^{+6.5}$ & $<6.1$ & $0.12_{-0.12}^{+0.42}$ & $<0.18$ \\
\hline $\mathrm{J} 132827.07+581836.9$ & 0405690201 & 2006-11-19 & 25.97 & pn & $27_{-167}^{+48.5}$ & $<27.6$ & $1.42_{-0.97}^{+1.03}$ & $<0.00$ \\
\hline $\mathrm{J} 133004.72+472301.0$ & 0112840201 & $2003-01-15$ & 17.11 & pn & $<12.3$ & $<7.9$ & $<0.69$ & $\ldots$ \\
\hline $\mathrm{J} 133553.61+514744.1$ & 0084190201 & $2002-06-12$ & 38.39 & pn & $86_{-24.3}^{+26.0}$ & $6_{-5.9}^{+21.2}$ & $2.40_{-0.84}^{+0.90}$ & $-0.87_{-0.13}^{+0.11}$ \\
\hline $\mathrm{J} 133639.40+514605.2$ & 0084190201 & $2002-06-12$ & 37.24 & pn & $<44.7$ & $<10.2$ & $<0.78$ & $\ldots$ \\
\hline J134145.12-003631.0 & 0111281601 & $2002-07-20$ & 7.41 & $\operatorname{mos} 1$ & $2_{-2.0}^{+5.3}$ & $<5.0$ & $0.23_{-0.23}^{+0.90}$ & $<0.40$ \\
\hline $\mathrm{J} 142555.22+373900.7$ & 0112230201 & $2002-12-18$ & 19.48 & pn & $29_{-11.2}^{+13.0}$ & $<28.6$ & $2.34_{-0.80}^{+0.23}$ & $<-0.01$ \\
\hline $\mathrm{J} 142539.38+375736.7$ & 0112230201 & $2002-12-18$ & 19.48 & pn & $109_{-22.5}^{+24.2}$ & $36_{-17.6}^{+19.4}$ & $7.44_{-1.49}^{+1.50}$ & $-0.50_{-0.20}^{+0.15}$ \\
\hline J142652.94+375359.9 & 0112230201 & $2002-12-18$ & 19.48 & pn & $14_{-13.2}^{+14.5}$ & $<32.9$ & $1.61_{-1.05}^{+1.15}$ & $<0.41$ \\
\hline
\end{tabular}


Table 2-Continued

\begin{tabular}{|c|c|c|c|c|c|c|c|c|}
\hline Name(SDSS) & Obs.ID ${ }^{\mathrm{a}}$ & Date & $T_{\exp }^{\mathrm{b}}$ & Instrument & $\mathrm{Soft}^{\mathrm{c}}$ & $\operatorname{Hard}^{\mathrm{c}}$ & Counts Rate ${ }^{\mathrm{d}}$ & $\mathrm{HR}^{\mathrm{e}}$ \\
\hline $\mathrm{J} 144027.00+032637.9$ & 0300210701 & 2006-01-08 & 23.05 & $\operatorname{mos} 1$ & $<19.5$ & $<17.7$ & $<1.08$ & $\ldots$ \\
\hline $\mathrm{J} 144625.48+025548.6$ & 0203050801 & 2005-01-12 & 7.86 & mos1 & $<3.1$ & $4_{-3.9}^{+5.4}$ & $0.13_{-0.13}^{+0.82}$ & $>0.13$ \\
\hline $\mathrm{J} 150824.22-000603.8$ & 0305750201 & $2005-07-20$ & 5.03 & $\operatorname{mos} 2$ & $6_{-4.5}^{+6.3}$ & $<11.3$ & $2.18_{-1.29}^{+-1.13}$ & $<0.34$ \\
\hline J152553.89+513649.1 & $0011830401(\mathrm{~T})$ & $2001-12-13$ & 2.82 & pn & $122_{-22.9}^{+2.5}$ & $56_{-169}^{+18.6}$ & $63.05_{-10.17}^{+10.86}$ & $-0.37_{-0.13}^{+0.12}$ \\
\hline J153229.97+323658.4 & 0039140101 & $2002-07-30$ & 4.74 & pn & $<8.7$ & $<11.7$ & $<2.97$ & $\ldots$ \\
\hline $\mathrm{J} 154359.44+535903.2$ & $0060370901(\mathrm{~T})$ & $2002-02-06$ & 16.18 & pn & $571_{-41.5}^{+43.1}$ & $115_{-22.9}^{+24.8}$ & $42.40_{-2.93}^{+3.06}$ & $-0.66_{-0.05}^{+0.04}$ \\
\hline J164151.84+385434.2 & 0204340101 & 2004-08-20 & 12.22 & pn & $<7.7$ & $<17.0$ & $<1.27$ & $\ldots$ \\
\hline
\end{tabular}

${ }^{a}(T)$ means the object is the intended PI target of the XMM-Newton observation.

${ }^{\mathrm{b}}$ The effective exposure time in $10^{3} \mathrm{~s}$.

${ }^{\mathrm{c}}$ Errors are $1 \sigma$ Poisson errors $($ Gehrels 1986$)$ for detections,and for non-detections the limits are the $90 \%$ confidence limits from Bayesian statistics (Kraft et al. 1991). The count rate is the full energy band,0.3-10.0keV.

d The count rate in $10^{-3}$ counts $s^{-1}$

${ }^{\mathrm{e}} H R$ is defined as $(h-s) /(h+s)$. The $H R$ errors are propagated from the counts errors using the Bayesian estimation of Park et al. (2006). Notice the difference of the definitions of the hard and soft band between ours $(s: 0.3-2.0 \mathrm{keV} ; h: 2.0-10.0 \mathrm{keV})$ and $\mathrm{G06s}(s: 0.5-2.0 \mathrm{keV} ; h: 2.0-8.0 \mathrm{keV})$ 
Table 3. X-RAY PROPERTIES

\begin{tabular}{|c|c|c|c|c|c|c|c|c|c|c|}
\hline Name(SDSS) & $N_{H}^{\mathrm{a}}$ & $\Gamma_{H R}^{\mathrm{b}}$ & $\log \left(f_{x}\right)^{\mathrm{c}}$ & $\log \left(f_{2 k e v}\right)^{\mathrm{d}}$ & $\log \left(f_{2500}\right)^{\mathrm{d}}$ & $\log \left(l_{2500}\right)^{\mathrm{e}}$ & $\alpha_{o x}$ & $\Delta \alpha_{o x^{\mathrm{f}}}^{\mathrm{f}}$ & $\alpha_{o x}(\operatorname{corr})^{\mathrm{g}}$ & $\Delta \alpha_{o x}(\operatorname{corr})^{\mathrm{h}}$ \\
\hline J020230.66-075341.2 & & & $<-13.418$ & $<-31.782$ & -27.140 & 30.726 & $<-1.78$ & $<-0.22$ & $<-1.34$ & $<0.22$ \\
\hline J023224.87-071910.5 & $2.96_{-1.14}^{+1.76}$ & $1.06_{-0.26}^{+0.32}$ & -13.230 & -31.547 & -26.861 & 30.945 & -1.80 & -0.21 & -1.57 & 0.02 \\
\hline J024304.68+000005.4 & $4.69_{-2.42}^{+4.34}$ & $1.12_{-0.22}^{+0.21}$ & -13.095 & -31.376 & -26.827 & 31.155 & -1.75 & -0.13 & -1.50 & 0.12 \\
\hline J085551.24+375752.2 & $0.58_{-0.56}^{+1.00}$ & $1.93_{-0.12}^{+0.52}$ & -13.576 & -31.331 & -26.794 & 31.162 & -1.74 & -0.12 & -1.73 & -0.11 \\
\hline J090928.50+541925.9 & & & $<-13.814$ & $<-32.178$ & -27.316 & 31.129 & $<-1.87$ & $<-0.25$ & $<-1.42$ & $<0.20$ \\
\hline J091127.61+055054.1 & $1.89_{-1.01}^{+1.58}$ & $1.22_{-0.11}^{+0.13}$ & -12.620 & -30.787 & -26.467 & 31.768 & -1.66 & $\ldots$ & -1.41 & $\ldots$ \\
\hline J091400.95+410600.9 & $\ldots$ & & $<-13.537$ & $<-31.902$ & -27.390 & 30.613 & $<-1.73$ & $<-0.19$ & $<-1.34$ & $<0.21$ \\
\hline J092138.45+301546.9 & $<21.25$ & $>0.21$ & -13.137 & -32.144 & -26.864 & 30.939 & $>-2.03$ & $>-0.44$ & $<-1.51$ & $<0.09$ \\
\hline J092238.43+512121.2 & $<0.01$ & $>2.38$ & -13.560 & -31.205 & -27.669 & 30.212 & $>-1.36$ & $>0.13$ & $<-1.47$ & $<0.03$ \\
\hline J092345.19+512710.0 & $<1.50$ & $>1.47$ & -14.155 & -32.167 & -27.177 & 30.869 & $>-1.92$ & $>-0.33$ & $<-1.43$ & $<0.16$ \\
\hline J092507.54+521102.6 & $<5.11$ & $>1.12$ & -13.463 & -31.721 & -26.852 & 31.433 & $>-1.87$ & $>-0.21$ & $<-1.56$ & $<0.10$ \\
\hline J094309.56+481140.5 & $\cdots$ & $\cdots$ & $<-13.436$ & $<-31.803$ & -27.049 & 30.856 & $<-1.83$ & $<-0.25$ & $<-1.45$ & $<0.14$ \\
\hline J094440.42+041055.6 & $\ldots$ & $\ldots$ & $<-13.571$ & $<-31.931$ & -26.861 & 31.116 & $<-1.95$ & $<-0.33$ & $<-1.53$ & $<0.09$ \\
\hline J095110.56+393243.9 & & & $<-13.651$ & $<-32.017$ & -27.518 & 30.346 & $<-1.73$ & $<-0.22$ & $<-1.39$ & $<0.13$ \\
\hline $\mathrm{J} 100728.69+534326.7$ & $2.39_{-1.19}^{+2.24}$ & $1.42_{-0.19}^{+0.24}$ & -13.131 & -31.206 & -27.268 & 30.621 & -1.51 & 0.04 & -1.37 & 0.18 \\
\hline $\mathrm{J} 105201.35+441419.8$ & $14.07_{-5.09}^{+8.61}$ & $0.89_{-0.33}^{+0.37}$ & -13.012 & -31.464 & -27.086 & 30.811 & -1.68 & -0.11 & -1.38 & 0.19 \\
\hline $\mathrm{J} 110853.98+522337.9$ & & & $<-12.834$ & $<-31.201$ & -27.065 & 30.775 & $<-1.59$ & $<-0.02$ & $<-1.38$ & $<0.19$ \\
\hline J111816.95+074558.1 & $0.36_{-0.06}^{+0.06}$ & $1.64_{-0.02}^{+0.02}$ & -12.228 & -30.155 & -25.995 & 31.877 & -1.60 & $\ldots$ & -1.51 & $\cdots$ \\
\hline $\mathrm{J} 112055.78+431412.5$ & $\ldots$ & $\ldots$ & $<-13.599$ & $<-31.963$ & -26.995 & 31.124 & $<-1.91$ & $<-0.29$ & $<-1.46$ & $<0.15$ \\
\hline $\mathrm{J} 112432.14+385104.3$ & $<5.48$ & $>1.03$ & -13.840 & -32.175 & -27.126 & 31.276 & $>-1.94$ & $>-0.30$ & $<-1.56$ & $<0.08$ \\
\hline $\mathrm{J} 113419.96+485805.7$ & $\ldots$ & $\ldots$ & $<-13.816$ & $<-32.182$ & -27.100 & 31.205 & $<-1.95$ & $<-0.32$ & $<-1.50$ & $<0.13$ \\
\hline $\mathrm{J} 113406.87+525959.0$ & $<45.73$ & $>-0.48$ & -13.158 & -32.835 & -27.123 & 30.765 & $>-2.19$ & $>-0.63$ & $<-1.37$ & $<0.20$ \\
\hline $\mathrm{J} 120449.77+020635.6$ & $\ldots$ & & $<-13.251$ & $<-31.616$ & -26.992 & 31.238 & $<-1.78$ & $<-0.14$ & $<-1.43$ & $<0.20$ \\
\hline $\mathrm{J} 120522.18+443140.4$ & $1.77_{-1.22}^{+2.50}$ & $1.16_{-0.24}^{+0.33}$ & -13.422 & -31.666 & -27.000 & 30.952 & -1.79 & -0.20 & -1.57 & 0.03 \\
\hline $\mathrm{J} 122708.29+012638.4$ & $<5.86$ & $>1.14$ & -13.842 & -32.099 & -27.272 & 30.694 & $>-1.85$ & $>-0.30$ & $<-1.56$ & $<0.00$ \\
\hline $\mathrm{J} 125741.41+565214.2$ & $<15.81$ & $>0.40$ & -13.959 & -32.827 & -27.361 & 30.558 & $>-2.10$ & $>-0.56$ & $<-1.44$ & $<0.10$ \\
\hline $\mathrm{J} 132827.07+581836.9$ & $<37.22$ & $>0.67$ & -13.540 & -32.228 & -26.904 & 31.415 & $>-2.04$ & $>-0.39$ & $<-1.53$ & $<0.13$ \\
\hline $\mathrm{J} 133004.72+472301.0$ & $\cdots$ & $\cdots$ & $<-14.098$ & $<-32.464$ & -27.140 & 31.103 & $<-2.04$ & $<-0.43$ & $<-1.64$ & $<-0.02$ \\
\hline $\mathrm{J} 133553.61+514744.1$ & $1.41_{-1.24}^{+3.70}$ & $2.56_{-0.45}^{+2.04}$ & -14.048 & -31.642 & -26.901 & 31.017 & -1.82 & -0.22 & -1.96 & -0.36 \\
\hline $\mathrm{J} 133639.40+514605.2$ & & & $<-14.084$ & $<-32.451$ & -27.239 & 30.828 & $<-2.00$ & $<-0.42$ & $<-1.67$ & $<-0.09$ \\
\hline J134145.12-003631.0 & $<37.45$ & $>-0.01$ & -13.475 & -32.773 & -26.899 & 31.163 & $>-2.26$ & $>-0.63$ & $<-1.43$ & $<0.20$ \\
\hline J142555.22+373900.7 & $<28.69$ & $>0.70$ & -13.413 & -32.058 & -27.108 & 31.110 & $>-1.90$ & $>-0.29$ & $<-1.44$ & $<0.17$ \\
\hline $\mathrm{J} 142539.38+375736.7$ & $2.90_{-1.17}^{+1.68}$ & $1.49_{-0.26}^{+0.46}$ & -13.246 & -31.267 & -26.826 & 31.117 & -1.71 & -0.09 & -1.57 & 0.05 \\
\hline J142652.94+375359.9 & $<31.55$ & $>0.04$ & -13.358 & -32.551 & -27.248 & 30.659 & $>-2.04$ & $>-0.48$ & $<-1.41$ & $<0.14$ \\
\hline
\end{tabular}


Table 3-Continued

\begin{tabular}{|c|c|c|c|c|c|c|c|c|c|c|}
\hline Name(SDSS) & $N_{H}^{\mathrm{a}}$ & $\Gamma_{H R}{ }^{\mathrm{b}}$ & $\log \left(f_{x}\right)^{\mathrm{c}}$ & $\log \left(f_{2 k e v}\right)^{\mathrm{d}}$ & $\log \left(f_{2500}\right)^{\mathrm{d}}$ & $\log \left(l_{2500}\right)^{\mathrm{e}}$ & $\alpha_{o x}$ & $\Delta \alpha_{o x}{ }^{\mathrm{f}}$ & $\alpha_{o x}(\text { corr })^{\mathrm{g}}$ & $\Delta \alpha_{o x}(\text { corr })^{\mathrm{h}}$ \\
\hline $\mathrm{J} 144027.00+032637.9$ & & $\ldots$ & $<-13.617$ & $<-31.980$ & -27.081 & 30.953 & $<-1.88$ & $<-0.29$ & $<-1.52$ & $<0.07$ \\
\hline $\mathrm{J} 144625.48+025548.6$ & $>13.56$ & $<0.56$ & -14.079 & -32.807 & -27.126 & 30.811 & $<-2.18$ & $<-0.61$ & -1.47 & 0.11 \\
\hline $\mathrm{J} 150824.22-000603.8$ & $<20.29$ & $>0.24$ & -12.869 & -31.846 & -27.030 & 30.767 & $>-1.85$ & $>-0.28$ & $<-1.32$ & $<0.24$ \\
\hline $\mathrm{J} 152553.89+513649.1$ & $5.36_{-3.28}^{+8.61}$ & $1.33_{-0.19}^{+0.22}$ & -12.556 & -30.636 & -26.028 & 32.230 & -1.77 & 0.00 & -1.55 & 0.22 \\
\hline $\mathrm{J} 153229.97+323658.4$ & & $\cdots$ & $<-13.615$ & $<-31.980$ & -27.179 & 31.119 & $<-1.84$ & $<-0.23$ & $<-1.39$ & $<0.22$ \\
\hline $\mathrm{J} 154359.44+535903.2$ & $1.11_{-0.35}^{+0.42}$ & $1.79_{-0.08}^{+0.12}$ & -12.889 & -30.684 & -26.330 & 31.783 & -1.67 & 0.03 & -1.63 & 0.08 \\
\hline $\mathrm{J} 164151.84+385434.2$ & & $\begin{array}{r}-0.08 \\
\cdots\end{array}$ & $<-13.961$ & $<-32.327$ & -26.444 & 32.004 & $<-2.26$ & $<-0.52$ & $<-1.73$ & $<0.00$ \\
\hline
\end{tabular}

a Intrinsic absorption column density in units of $10^{22} \mathrm{~cm}^{-2}$,its value or upper or lower limit is determined by fitting the observed spectrum,or comparing the observed HR to a simulated HR that takes the instrument response into account,or the upper limit of count rates,assuming $\Gamma=2.0$ and a simple neutral absorption.

${ }^{\mathrm{b}} \Gamma_{H R}$, following the definition of G06s, is a coarse measure of the hardness of the X-ray spectrum determined by comparing the observed HR to a simulated HR that takes the instrument response into account.

${ }^{\mathrm{c}}$ The full-band X-ray flux, $f_{\mathrm{x}}$, has units of $\mathrm{erg} \mathrm{cm}^{-2} \mathrm{~s}^{-1}$.

dX-ray and optical flux densities were measured at rest-frame $2 \mathrm{keV}$ and $2500 \AA$, respectively; units are $\operatorname{erg~cm}^{-2} \mathrm{~s}^{-1} \mathrm{~Hz}^{-1}$.

e The $2500 \AA$ monochromatic luminosity, $l_{2500}$, has units of $\mathrm{erg} \mathrm{s}^{-1} \mathrm{~Hz}^{-1}$. The redshift bandpass correction has been included.

${ }^{\mathrm{f}}$ The parameter $\Delta \alpha_{\mathrm{ox}}$ is the difference between the observed $\alpha_{\mathrm{ox}}$ and $\alpha_{\mathrm{ox}}\left(l_{2500}\right)$, the predicted $\alpha_{\mathrm{ox}}$ from $l_{2500}$ calculated from Equation 6 of $($ Strateva et al. 2005).

${ }^{\mathrm{g}}$ The parameter $\alpha_{\mathrm{ox}}$ (corr) is $\alpha_{\mathrm{ox}}$ calculated assuming $\Gamma=2.0$ and using the hard-band count rate to normalize the X-ray continuum.

${ }^{\mathrm{h}} \Delta \alpha_{\mathrm{ox}}(\operatorname{corr})=\alpha_{\mathrm{ox}}(\operatorname{corr})-\alpha_{\mathrm{ox}}\left(l_{2500}\right)$. 
Table 4. Results from Non-Parametric Bivariate Statistical Tests

\begin{tabular}{|c|c|c|c|c|}
\hline $\begin{array}{c}\text { Variables }^{\mathrm{a}} \\
\text { Independent/Dependent }\end{array}$ & $\begin{array}{c}\text { Gener } \\
\tau\end{array}$ & $\begin{array}{l}\text { ized Kendall } \\
\text { Prob. }^{\mathrm{b}}(\%)\end{array}$ & $\rho$ & $\begin{array}{l}\text { Spearman } \\
\text { Prob. }^{\mathrm{b}}(\%)\end{array}$ \\
\hline$N_{\mathrm{H}} / \Delta \alpha_{\mathrm{ox}}(51)$ & 6.930 & $<0.01$ & $\ldots$ & . \\
\hline$N_{\mathrm{H}} / \Delta \alpha_{\mathrm{ox}}(\operatorname{cor} r)(51)$ & 0.913 & 36.2 & $\ldots$ & .. \\
\hline$N_{\mathrm{H}} / \mathrm{BI}(53)$ & 2.296 & 2.2 & $\cdots$ & .. \\
\hline$N_{\mathrm{H}} / v_{\max }(53)$ & 0.365 & 71.5 & $\cdots$ & .. \\
\hline$\Delta \alpha_{\mathrm{ox}} / \mathrm{BI}(74)$ & 3.467 & 0.05 & $\ldots$ & .. \\
\hline$\Delta \alpha_{\mathrm{ox}} / v_{\max }(74)$ & 2.003 & 4.5 & $\cdots$ & $\cdots$ \\
\hline$\Delta \alpha_{\mathrm{ox}} / \mathrm{BI}(\mathrm{HiBALs}$ only 45$)$ & 2.553 & 1.1 & $\ldots$ & .. \\
\hline$\Delta \alpha_{\text {ox }} / v_{\max }($ HiBALs only 45$)$ & 0.414 & 67.9 & $\cdots$ & $\cdots$ \\
\hline$\alpha_{\mathrm{ox}}(\operatorname{corr}) / \mathrm{BI}(74)$ & 3.657 & 0.03 & -0.384 & 0.1 \\
\hline$\alpha_{\mathrm{ox}}(\operatorname{corr}) / v_{\max }(74)$ & 3.936 & 0.01 & -0.458 & 0.01 \\
\hline$\alpha_{\text {ox }}($ corr $) / \mathrm{BI}($ HiBALs only 45$)$ & 3.447 & 0.06 & -0.492 & 0.1 \\
\hline$\alpha_{\text {ox }}(\operatorname{corr}) / v_{\max }($ HiBALs only 45$)$ & 3.335 & 0.09 & -0.469 & 0.2 \\
\hline$\Delta \alpha_{\mathrm{ox}}($ corr $) / \mathrm{BI}(74)$ & 3.215 & 0.1 & -0.329 & 0.5 \\
\hline$\Delta \alpha_{\mathrm{ox}}(\operatorname{corr}) / v_{\max }(74)$ & 3.623 & 0.03 & -0.425 & 0.03 \\
\hline$\Delta \alpha_{\mathrm{ox}}(\operatorname{corr}) / \mathrm{BI}(\mathrm{HiBALs}$ only 45$)$ & 3.071 & 0.2 & -0.430 & 0.4 \\
\hline$\Delta \alpha_{\mathrm{ox}}(\operatorname{corr}) / v_{\max }($ HiBALs only 45$)$ & 3.218 & 0.1 & -0.473 & 0.2 \\
\hline
\end{tabular}

aThe number of data points is given in parentheses.

b The probability(in units of percent) that the given variables are not correlated. Spearman's $\rho$ cannot be calculated for data with both upper and lower limits. 


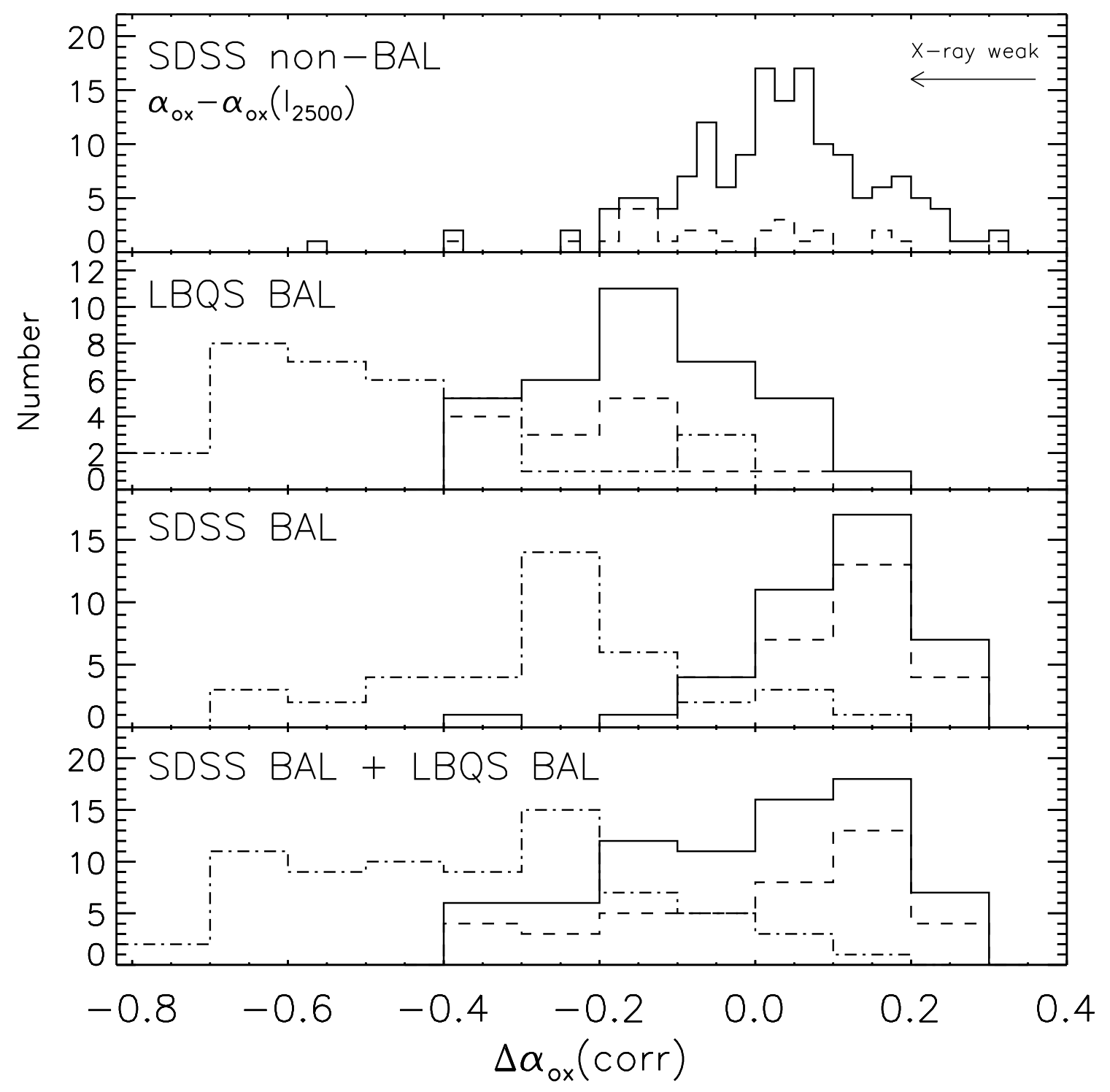

Fig. 1. - The top panel shows the distribution of observed $\Delta \alpha_{\mathrm{ox}}=\alpha_{\mathrm{ox}}-\alpha_{\mathrm{ox}}\left(l_{2500}\right)$ for the SDSS / ROSAT non-BAL sample(Strateva et al. 2005). The three lower panels show the distributions of $\Delta \alpha_{\text {ox }}($ corr $)=\alpha_{\text {ox }}($ corr $)-\alpha_{\text {ox }}\left(l_{2500}\right)$ for the LBQS/Chandra BAL sample(G06), the SDSS/XMM-Newton BAL sample and the combined sample(this paper),respectively.For all four panels,solid lines indicate the full samples and dashed lines only show upper limits.Dotdashed line in the three lower panels represents the distribution of $\Delta \alpha_{\text {ox }}$. The arrow in the top panel shows the direction of the X-ray weak objects for our convention of $\alpha_{\mathrm{ox}}$. 

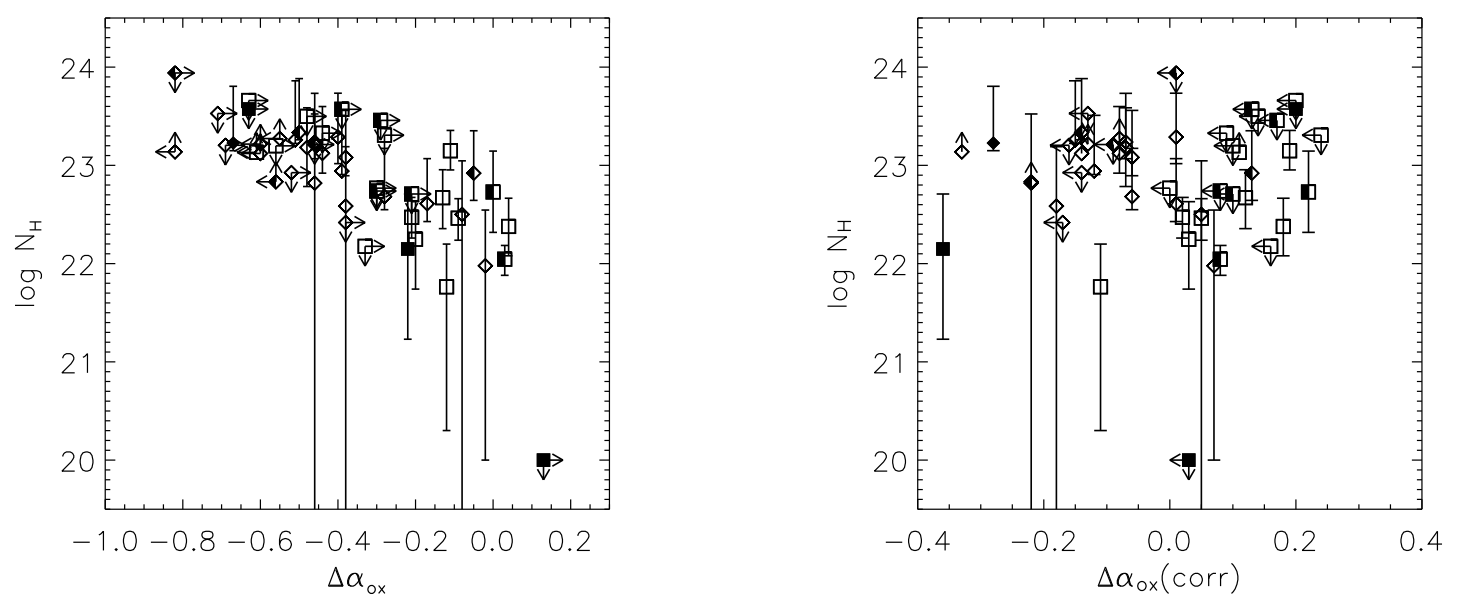

Fig. 2.- The left panel shows the $N_{\mathrm{H}}$ vs. $\Delta \alpha_{\mathrm{ox}}=\alpha_{\mathrm{ox}}-\alpha_{\mathrm{ox}}\left(l_{2500}\right)$ and the right panel shows the $N_{\mathrm{H}}$ vs. $\Delta \alpha_{\text {ox }}($ corr $)=\alpha_{\text {ox }}($ corr $)-\alpha_{\text {ox }}\left(l_{2500}\right)$ for the detected BAL QSOs in the combined sample.SDSS BAL QSOs in our sample are shown with squares and LBQS BAL QSOs in G06's sample are shown with diamonds. The open,filled and half-filled symbols indicate HiBALs,LoBALs and BAL QSOs of unknown type,respectively.
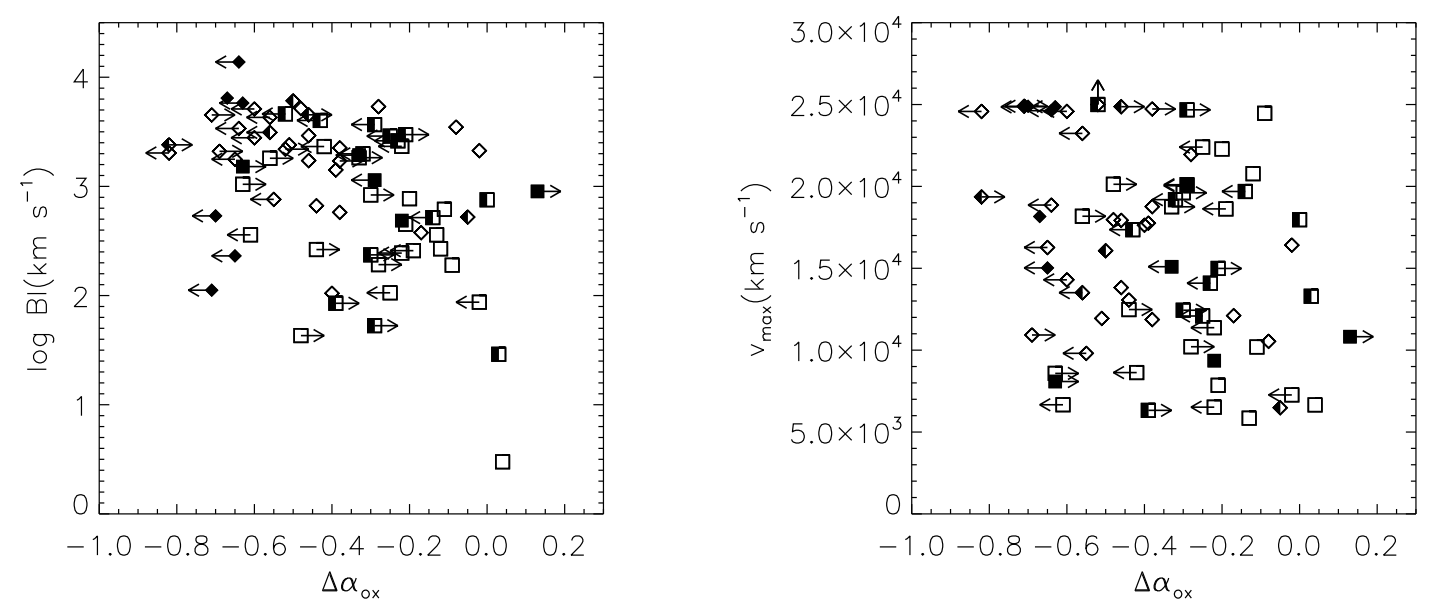

Fig. 3.- Plots of $\Delta \alpha_{\mathrm{ox}}=\alpha_{\mathrm{ox}}-\alpha_{\mathrm{ox}}\left(l_{2500}\right)$ vs. C IV absorption-line parameters for the combined sample : BALnicity index(BI, left panel) and maximum outflow velocity of absorption, $\left(v_{\max }\right.$, right panel).Symbols are the same as in Fig. 2. 

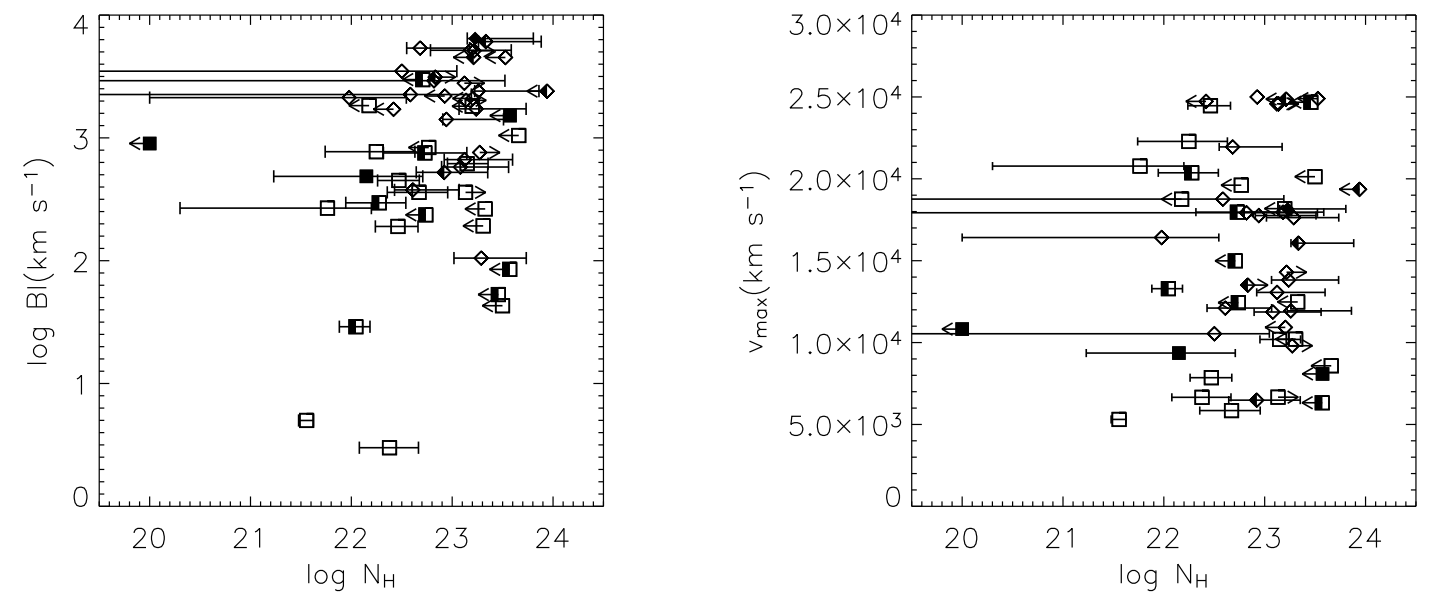

Fig. 4.- Plots of $N_{\mathrm{H}}$ vs. C IV absorption-line parameters for the detected BAL QSOs in the combined sample : BALnicity index(BI, left panel) and maximum outflow velocity of absorption, $\left(v_{\max }\right.$, right panel).Symbols are the same as in Fig. 2] .
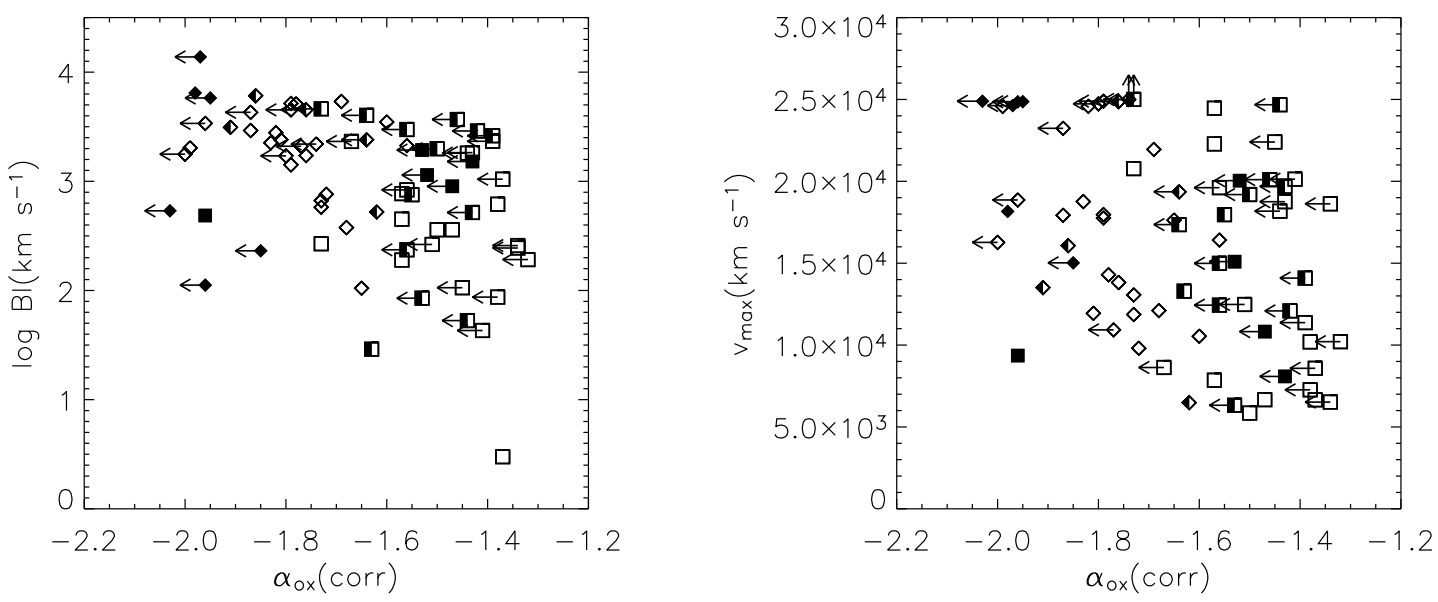

Fig. 5.- Plots of $\alpha_{\text {ox }}$ (corr) vs. C IV absorption-line parameters for the combined sample : BALnicity index(BI, left panel) and maximum outflow velocity of absorption, $\left(v_{\max }\right.$, right panel).Symbols are the same as in Fig. 2 . 

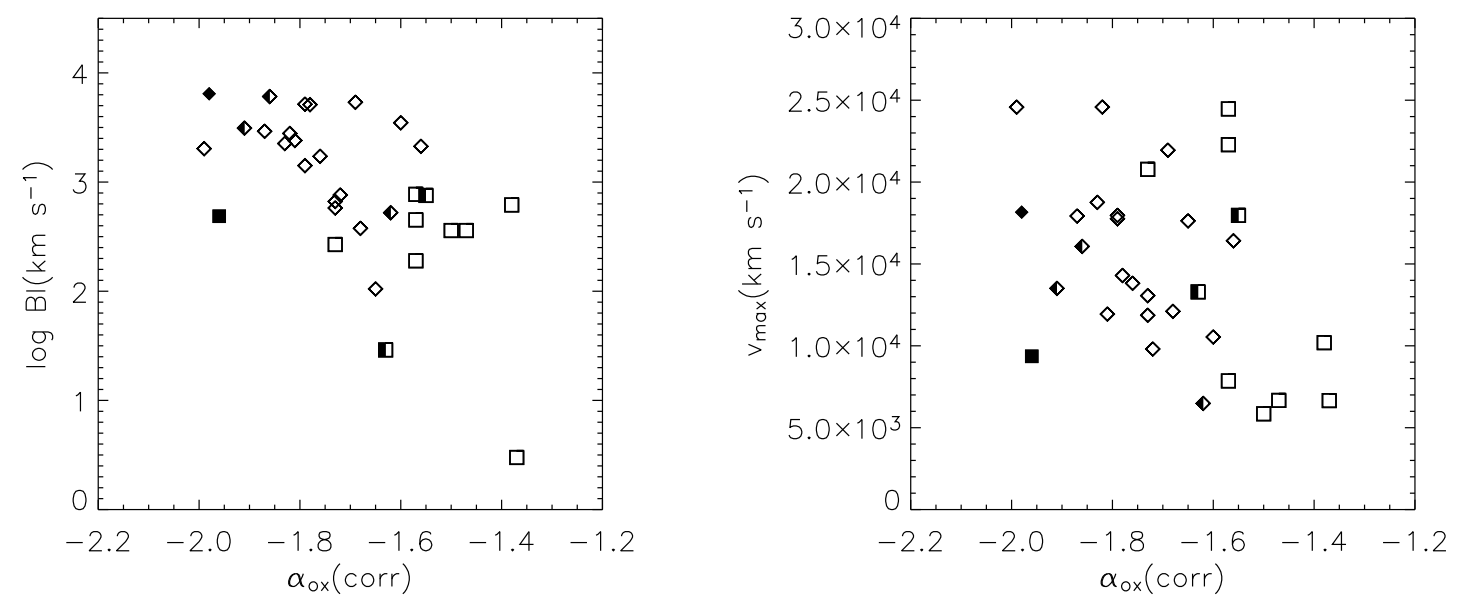

Fig. 6.- Plots of $\alpha_{\text {ox }}$ (corr) vs. C IV absorption-line parameters for the BAL QSOs detected in the hard X-ray band in the combined sample: BALnicity index(BI, left panel) and maximum outflow velocity of absorption, $\left(v_{\max }\right.$, right panel).Symbols are the same as in Fig. 2 . 